\title{
Quartz Crystal Microbalance as a Sensor to Characterize Macromolecular Assembly Dynamics
}

\author{
K. Kanazawa ${ }^{1}$ and Nam-Joon Cho ${ }^{2}$ \\ ${ }^{1}$ Department of Chemical Engineering, Stanford University, Stanford, CA 94305, USA \\ ${ }^{2}$ Division of Gastroenterology, School of Medicine, Stanford University, Stanford, CA 94305, USA
}

Correspondence should be addressed to K. Kanazawa, procmon@msn.com

Received 22 December 2008; Accepted 10 April 2009

Recommended by Michele Penza

The quartz crystal microbalance sensor has a resonant frequency $f$ and a quality factor $Q$ which can be used to probe the properties of nanometer thick film loads. A recent review by Arnau (2008) has discussed many of the considerations necessary to accurately probe for these properties. To avoid needless duplication but to still provide an adequate background for the new user, we briefly outline the basic measurement methodologies and analytical techniques that were covered in the review. Details will be provided on some specific perspectives of the authors. For example, the special precautions necessary when dealing with soft films (polymeric and biological) under liquid are overviewed. To illustrate applications of the QCM technique, simple bilayer and vesicle behaviors are discussed, along with the structural transformation resulting from protein adsorption onto an intact vesicle adlayer. The amphipathic $\alpha$-helical (AH) peptide interaction is given as a particular example. Lastly, we summarize a top-down approach to functionalize a surface with a cell membrane and to study its interaction with proteins.

Copyright (c) 2009 K. Kanazawa and N.-J. Cho. This is an open access article distributed under the Creative Commons Attribution License, which permits unrestricted use, distribution, and reproduction in any medium, provided the original work is properly cited.

\section{Introduction}

The transducer for the quartz crystal microbalance (QCM) sensor is often in the form of a piezoelectric circular disk. Many aspects of piezoelectric films used as sensors are discussed in some detail in a recent book [1]. The general form of the common AT-cut quartz resonator is illustrated below in Figure 1.

There are two electrodes on the crystal's opposing faces. Upon an excitation (mechanical or electrical), thickness shear acoustic waves can undergo constructive interference such that resonances occur at particular frequencies. These are discussed in the classical book by $\mathrm{Lu}$ and Czanderna [2], an excellent review article related to the QCM in electrochemistry by Buttry and Ward [3], and in an oft-quoted text by Bottom [4]. Two modes of operation are possible. The resonances can be observed either under steady-state conditions (such as under a steady applied radio frequency potential) or under transient conditions (such as a decay following an initial excitation). For both cases, it has been established that a very accurate equivalent circuit describing the electrical behavior in the neighborhood of a resonance is given by the Butterworth Van Dyke (BVD) circuit as shown in Figure 2 and described by Cernosek et al. [5], and Muramatsu et al. [6].

The utility of the BVD circuit lies in the fact that the behavior of the resonator is generally probed using electrical signals. The physical behavior of the QCM is summarized in the elements of the BVD circuit. There are four elements. The branch on the left consists of a single element, the parallel capacitance $C_{P}$, which is simply the dielectric capacitance of the disc and can also reflect any external capacitances that occur when connecting the QCM to instrumentation. It does not reflect the motion of the disc and is a constant. The branch on the right is the so-called motional branch and consists of three components: the inductance $L$, a series capacitance $C_{S}$, and a series resistance $R$, which reflects the mechanical losses in the resonant system. These elements are sensitive to the disc's motion. The changes in these elements under loading of the quartz crystal by a liquid and/or a film are sensitive to the properties of the load. The series resonant frequency $f_{0}$ of $L$ and $C_{S}$ defines the resonant frequency and 


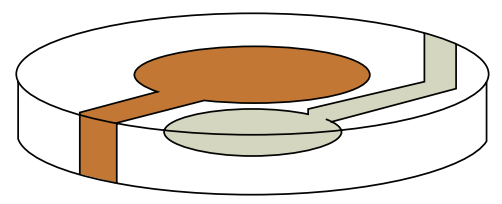

FIGURE 1: A piezoelectric disc forming the resonant transducer for the QCM.

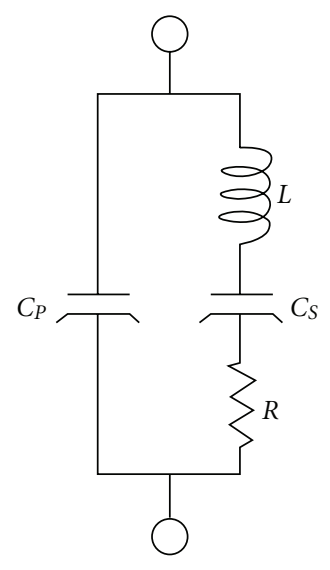

Figure 2: The butterworth van dyke electrical equivalent circuit near a resonance.

$R$ characterizes the losses. Both of these are sensitive to the load properties as examined by Martin et al. [7], and Bandey et al. [8].

\section{Measurement Methods}

As mentioned earlier, it is possible to use both steady-state and transient methods to experimentally characterize the loaded resonator. We begin by discussing the steady-state methods: the oscillator system and the impedance analysis system.

The oscillator is an electronic amplifying circuit in which the transducer serves as an active element through which positive feedback is applied such that the system oscillates. When the parallel capacitance $C_{P}$ can be neglected, and where the amplifier is nearly ideal, the circuit will oscillate at the zero-phase resonant frequency defined by the series resonance of $L$ and $C_{S}$. But as illustrated in Figure 2, we see that $C_{P}$ introduces an additional phase shift which can change the resonant frequency from its ideal value. A number of methods to correct for $C_{P}$ have been previously discussed in detail [9]. The user needs only to recognize that this correction is necessary and to ensure that precautions are taken to remove the effect of $C_{P}$ in their instrumentation. From the oscillator circuit, both the resonant frequency and the load loss are measured. This load loss can be related to the amount of feedback necessary to maintain oscillation or from the value of the current at resonance.

It is possible to excite the resonator not only at its fundamental frequency but at its odd harmonics [10]. The work, to be discussed later in this section, has shown that the study of the harmonics can add valuable additional information about the film's properties. A particularly fine example of this work was recently done by Vogt et al. [11]. One of the primary values is to characterize the change in the resonant frequency under film load with the harmonic variation. If the resonant frequency change due to the film load is proportional to the harmonic number $(1,3,5,7,9$, etc.), this can be taken as some evidence that the film can be treated as a rigid film. This infers the applicability of the Sauerbrey relation [12] where the resonant frequency at a given resonance decreases linearly with increasing mass load. This simplification can be very useful in many cases as has been discussed, for example, by Rodahl et al. [13], and by Voinova et al. [14]. If this relation is not satisfied, it indicates that a more complex analysis of the mass loading and the viscous loss effects of the load must be considered.

In general, the oscillator circuits to date have not had the capability of measuring at multiple harmonic frequencies, although switching networks have been utilized to make this possible recently. Recently, a dual harmonic oscillator (DHO), which operates at the fundamental and the third harmonic has been developed [15] which was inspired by the studies showing such a possibility [16]. In the DHO, the crystal with its load is excited simultaneously at two harmonic frequencies, for example, the first and the third. The distorting influence of $C_{P}$ has been removed, and both resonant frequencies and resistances at the two harmonic frequencies are measured. This simultaneous measurement of the same crystal (and therefore under identical conditions) is unique.

The second major steady-state method is that of impedance analysis. In this case, an rf voltage is applied across the crystal and is scanned over the region of the resonance of interest. Such methods are discussed, for example, by Kipling and Thompson [17], and by Lucklum et al. [18]. The QCM serves as a passive device under test. The ratio of the voltage to the current yields the electrical impedance of the device. Most applications of this scanning technique use the electrical admittance instead of the impedance. The admittance is simply the inverse of the impedance. The measurements taken are both the magnitude and phase of the admittance at each frequency or the real and imaginary parts of the admittance (the conductance and the susceptance). The use of the Butterworth Van Dyke circuit shown in Figure 2 is extremely useful in fitting the measurements. Typical conductance versus frequency and susceptance versus frequency curves are depicted in Figure 3. These curves were calculated using a one-dimensional model for lightly loaded resonator, and yield the conductance and susceptance in Siemens $\mathrm{m}^{-2}$.

The conductance is a maximum, $G_{\max }$, at the resonant frequency; the value of $G_{\max }$ yields the inverse of the resistance $R$. From $B_{\max }$ and $B_{\min }$, the value of $C_{p}$ can be determined.

The methodogies for obtaining these values are discussed in $[17,18]$. As is so often the case, there are possible effects, which can distort the spectrum and result in inaccurate fittings. Two of the main sources of these effects are discussed in an article in this issue [19]. We can determine $C_{P}$ from the 


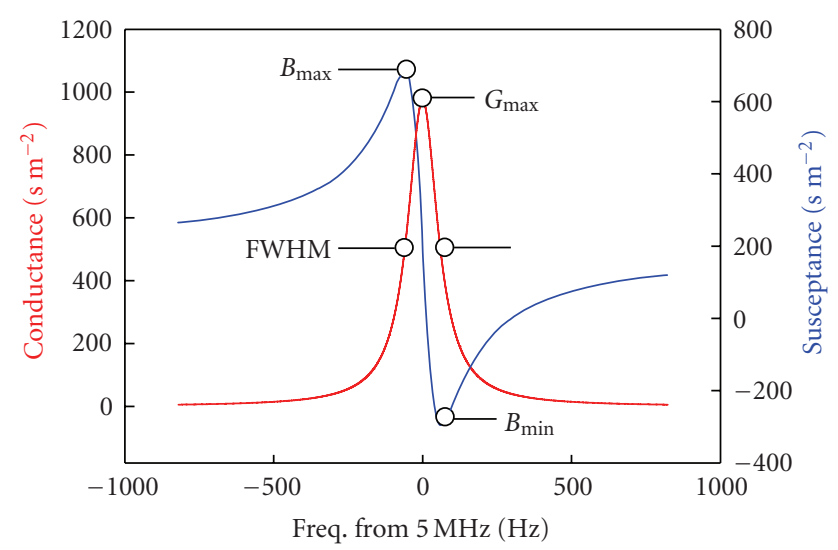

Figure 3: The conductance (red) and the susceptance (blue) versus the frequency from resonance are shown. Also, key points used for analysis are indicated.

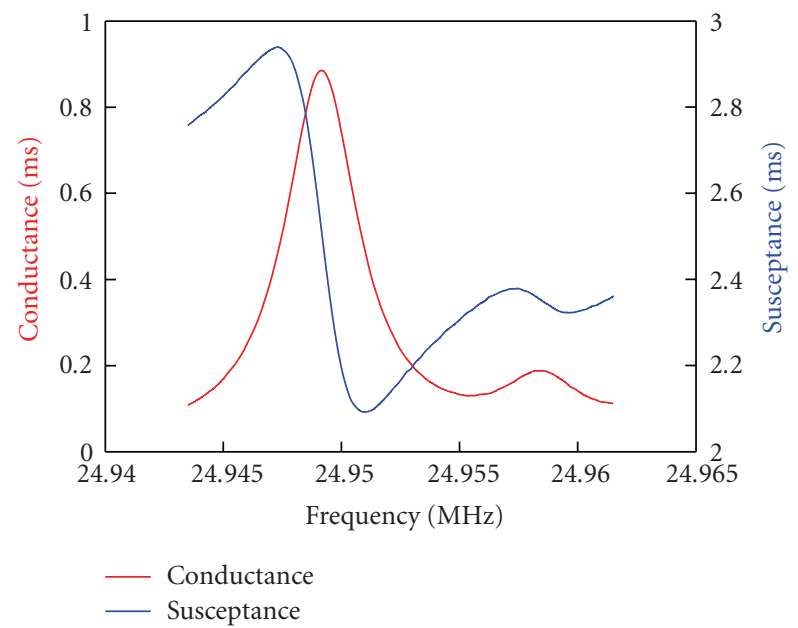

Figure 4: A spurious resonance (Spur) is shown lying above the main resonance of the 5 th harmonic of a $5 \mathrm{MHz}$ crystal. This is experimental data on a crystal in water with an area of $3.419 \times$ $10^{-5} \mathrm{~m}^{-2}$. The conductance and susceptance are in milliSiemens.

spectrum above. In addition to the contribution of a shunt cabling to $C_{P}$, a shunt conductance can also be introduced via external cabling. This can be recognized and corrected. The effects of these shunting elements can be corrected for through the measurements. Finally, spurious resonances (spur) that lying a few kilohertz above the resonance can be excited and can distort the spectrum. We have also shown that this effect can be eliminated by specifically and quantitatively accounting for its effects. An example of the presence of a spur resonance is shown in Figure 4 as revealed by experimental data.

The main resonance is seen to occur at about $24.95 \mathrm{MHz}$ but a spur is seen near $24.959 \mathrm{MHz}$. Its spectrum overlaps the main resonance spectrum, distorting it. This overlap can be removed by accounting for the spur using a separate resonance. The main resonance is then described accurately by a Lorentzian. From the fitting of the main resonance to the Lorentzian curve described by the Butterworth Van Dyke

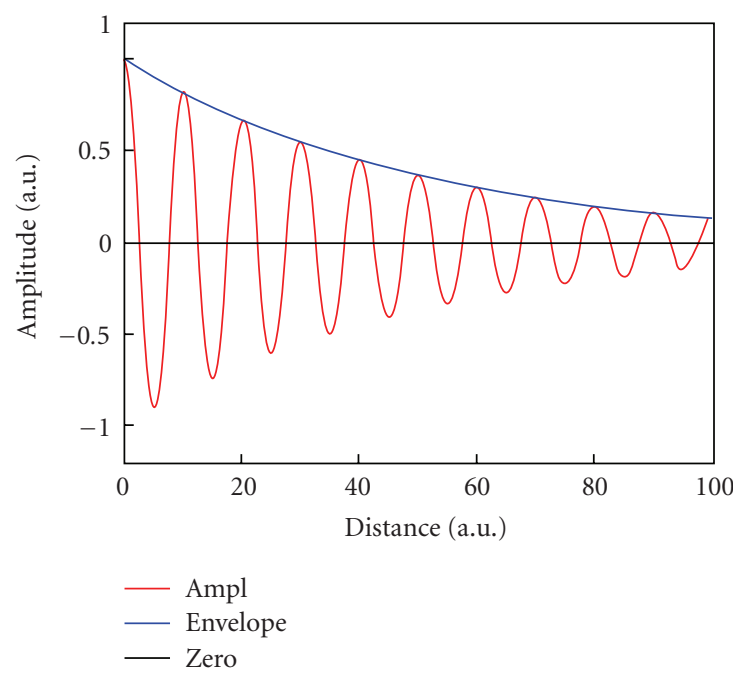

Figure 5: The current decay after excitation is suggested by the red curve.

circuit, the values of the elements of the circuit $\left(L, C_{S}, R\right.$, and $C_{P}$ ) can be determined.

A very different approach was taken at Chalmers Institute of Technology where they used transient decay of a crystal to determine the properties of the loading film and liquid [20]. In a subsequent work, they focused on the transient current decay resulting from a short circuit analysis [13]. The frequency of the applied voltage is varied until reaching the frequency where the largest current is observed. The crystal is then shorted, leaving the current to decay. The cartoon in Figure 5 suggests the transient behavior of the current with time.

The red curve indicates the current decay after initial excitation. The period of the resonant frequency is given by the spacing between the peaks or by the alternate zero crossing of the current. The quality of the resonance is given by the decay time of the envelope, shown in the blue. The horizontal black line is the zero line shown for reference. The resonant frequency and the quality $(Q)$ factor, or its inverse the dissipation $D$, can be measured for the resonator.

If the QCM is loaded with a lossy layer, then the envelope will decay more rapidly. The quality of the resonance can be related to the decay time. The details on how the films properties can be obtained from the resonant frequency and $Q$ are given in [13]. In a commercialized system, it has been possible to collect data from a number of the odd resonances $(1,3,5$, and 7$)$ and also $(1,3,5,7,9$, and 11$)$.

\section{Analytical Methods}

In order to relate the changes in the behavior of the resonance to the properties of the load, it is necessary to employ a quantitative model. A number of these exist, and most of them are based on a one-dimensional analysis of the resonator and load as will be discussed in a later section. The model assumes a disc of infinite lateral extent, with the only dimensional variable being along the direction perpendicular 


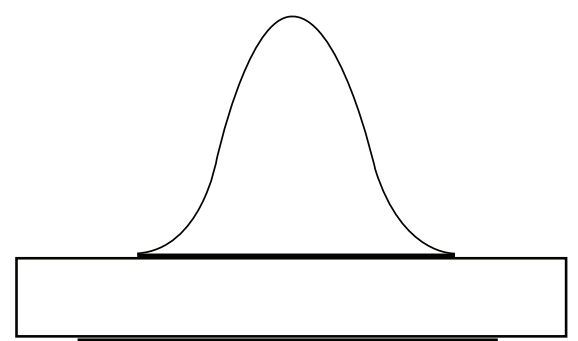

FIgURE 6: The distribution of the displacement amplitude is shown. The quartz and the electrodes are represented the rectangle and the dark lines, respectively.

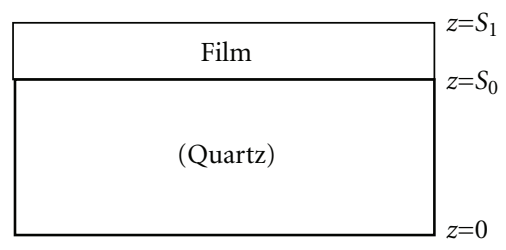

FIGURE 7: Mechanical representation of the quartz resonator loaded with a film.

to the disc. The actual behavior of the resonator is not one-dimensional however, but methods have been used to minimize this effect. This is briefly summarized in Figure 6.

The displacement of the device is not uniform over the surface $[21,22]$ and has a maximum at the center and decreases towards the electrode edges. In fact, this effect can be used for specific purposes such as rupture event scanning [23] or for binding perturbations studies [24]. For general cases, the one-dimensional models have been found to be quite adequate for the determination of load properties. The radial sensitivity function is mentioned here only to acquaint the user of that behavior. Its effect will be ignored in this presentation.

There are a large number of methods for analyzing the measurements such that the properties of the load on the QCM can be extracted. A number of different approaches have been taken. They include the purely mechanistic treatment where the acoustic resonances with the load in a purely mechanical manner, description in terms of mechanicalelectrical analogs using the Butterworth Van Dyke circuit, transmission line analogs for the sensor, and a variety of simplifications based on linearizing the results from the more complex solutions. A full electromechanical analysis has been presented, which will be discussed in more detail at the end of the paper.

The initial analyses treated the acoustomechanical resonances of the slab of quartz. This view of the resonator can be summarized as in Figure 7.

Early studies treated the quartz and film as lossless. A steady-state sinusoidal acoustic shear vibration at a frequency $f$ was assumed. The frequency $f$ is a real quantity. Sauerbrey had in mind such a model when for thin elastic films he understood that the film mass could then be approximated as an additional quartz mass. This resulted in a linear relation between the decrease in resonant frequency and the mass loading of the film with a proportionality constant dependent only on the quartz parameters. This has been of extreme value in the measurement of vapor deposited flms in vacuum.

The linear behavior was valid for approximately $2 \%$ or less change in frequency. The range of quantitative relation between the resonant frequency and the mass loading can be extended in range if one accounts for the acoustic impedance of the film, which can be expressed in terms of the film density $\rho$ and shear modulus $\mu$

$$
Z=\sqrt{\rho \mu} .
$$

The multiple reflections at the surfaces at $S=0, S=S_{0}$, and $S=S_{1}$ were summed and conditions for resonance determined [25]. This was put into a clean usable form by $\mathrm{Lu}$ and Lewis [26] and has proved very useful in extending the range of use of the QCM in a quantitative manner, although the simple linear functionality was lost. The curvature of the frequency-mass relation that was dependent on the material's property. However, the analysis was still restricted to lossless films.

Nomura and Minemura found that it was possible to use the QCM under liquid [27]. The liquid is viscous and introduces a loss into the system. In Figure 7, the liquid can be represented as an additional infinitely thick layer above the film. The relation between the frequency change and the liquid was found to be able to be described by the real part of a complex frequency as demonstrated in [28]. A complex frequency was necessary because of the existence of losses which precluded a steady-state solution under purely mechanical excitation. That is, a general time dependence of the type $e^{j \omega t}$ is assumed. If the angular frequency $\omega$ is complex, it can be seen that the real part gives a cycling sinusoid, while the imaginary part yields a time-decaying envelope. The general method used to solve for the resonance of such a layered system is to assign amplitude magnitudes to the acoustic waves traveling in the $+z$ direction and in the $-z$ direction. These acoustic waves are then required to satisfy the boundary conditions at the interfaces.

Prodded by the observation by Behling et al. [29], that the equations satisfying these boundary conditions can be separated into separate terms involving the mass and the viscoelastic properties of the load, it has been possible to obtain solutions using the complex frequency notion with the purely mechanical open circuit analysis to separate the mass loading from the viscoelastic parameters of the load. The separation into a mass dependent expression and a viscoeleastic dependent expression permits some very useful simplifications under certain conditions. This description is not discussed further here, but is presently under preparation.

A variant of the mechanical model was used by the Chalmers group [30] to quantify the relation between the resonant frequency and the mass loading and viscoelasticity. They concentrated on the load, treating the quartz as a rigid body, as suggested in Figure 8.

By including the possibility of a steady-state external excitation such as by an externally applied voltage from a 


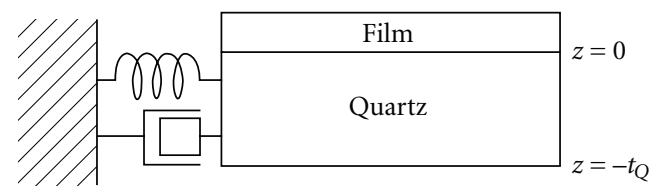

FIGURE 8: The configuration used where the quartz, shown by the open bottom rectangle, is treated as rigid and the effects of the load above it are summarized as changes in the spring constant and dashpot to the left of the quartz. Recall that the QCM under investigation is shorted. Therefore, the shunting impedances are shorted out and can be neglected.

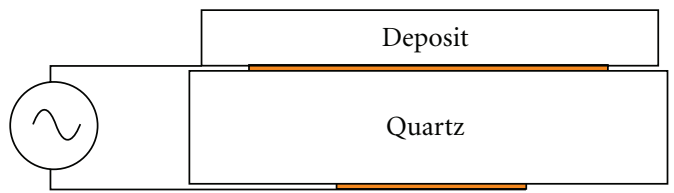

FIGURE 9: Electrodes at the bottom of the quartz and at the quartzfilm interface allow the application of an rf voltage.

voltage source as suggested in Figure 9, it is possible to move to a complete solution under those conditions.

This ability to apply an external steady-state voltage made possible a number of different ways to analyze the resonator with a load. Again however, the acoustic waves in each media are assigned amplitudes and the boundary conditions at the interfaces need to be satisfied. In fact, if the model is analyzed using the physical parameters including the piezoelectric properties of the quartz, then it is possible to show that the electrical impedance can be represented by a transmission line equivalent circuit as shown in Figure 10 [31]. This equivalent circuit has been particularly useful to show further simplifications following simplifications. It is not possible to do a complete description of the transmission line approach here. It is hoped however, to show that the specific piezoelectric and dielectric properties of the quartz crystal are specifically taken into account in this analysis. More detailed treatments are to be found in $[5,18,31]$. The influences of the quartz piezoelectric constant and dielectric constant are shown in the specific elements representing the piezoelectric driving circuitry in the transmission line. A voltage driving source applies a voltage $V$ to the circuit. The coupling to the quartz is represented by three elements, a capacitance $C_{0}$, a reactive element $j X$, and a transformer with the turns ratio of $1: N$ :

$$
\begin{aligned}
C_{0} & =\frac{\varepsilon_{22} A}{d_{Q}}, \\
X & =\frac{e_{26}^{2}}{\varepsilon_{22}^{2} \omega^{2} Z_{Q} A} \sin \left(k_{Q} d_{Q}\right), \\
N & =\frac{\sqrt{A}}{\left(2 e_{26} / \varepsilon_{22} \omega Z_{Q}\right) \sin \left(d_{Q} / 2\right)} .
\end{aligned}
$$

The variable $A$ here is the effective area of the resonator, usually being the area of the smaller electrode. $d_{Q}$ is the

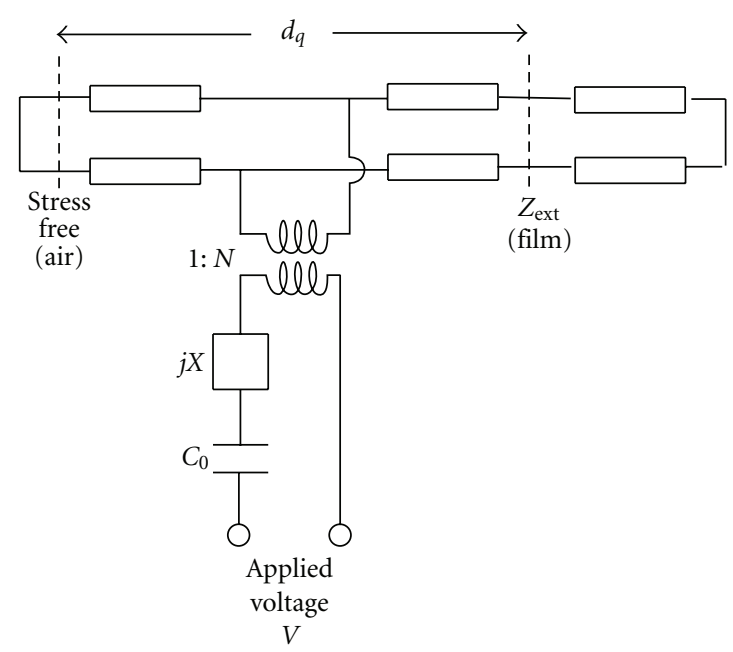

FIGURE 10: The representation of the transmission line equivalent circuit which serves as starting point for many useful simplifications. It includes the electric and piezoelectric properties of the quartz.

thickness of the resonator being about $3.3 \times 10^{-4} \mathrm{~m} . \varepsilon_{22}$ is the dielectric constant of the quartz having the value of $3.982 \times 10^{-11} \mathrm{fd} / \mathrm{m}$. $\omega$ is the angular frequency. $Z_{Q}$ is the acoustic impedance of the quartz which for loss less quartz is given by

$$
Z_{Q}=\sqrt{\rho_{Q} \overline{\mathcal{C}}_{66}}
$$

where

$$
\bar{c}_{66}=c_{66}+\frac{e_{26}^{2}}{\varepsilon_{22}} .
$$

Here $c_{66}$ is the appropriate mechanical shear modulus having the value of $2.901 \times 10^{10}$ Pascals, $e_{26}$ is the piezocoefficient having the value of -0.095 Coulombs $\mathrm{m}^{-2}$. $r_{\mathrm{Q}}$ is of course the quartz density in $\mathrm{kgm} \mathrm{m}^{-3} \cdot k_{Q}$ is the propagation constant for the shear waves in the quartz and is given by the relation

$$
k_{Q}=\omega \sqrt{\frac{\rho_{Q}}{\bar{c}_{66}}} .
$$

The section shown between the dashed lines represents the quartz and the right and left connections represent the interfaces of the quartz. We have portrayed the case where the crystal is loaded only on one face. The left face is left free so that the stress applied to that interface is zero. The electrical equivalent is a short circuit, as shown. On the right is the acoustic load provided by a film. The external surface of the film is assumed to be unloaded and is represented again by a short circuit. The various rectangular elements represent an electrical equivalent of the acoustic delay paths, giving rise to phase shifts across the elements. The general quartz elements do not change with load. Further simplifications of the analysis can be made under conditions where the resonance has a high $Q$ factor.

Of many simplifications, one of the most useful ones was proposed by the Sandia group [10]. They have shown that the 


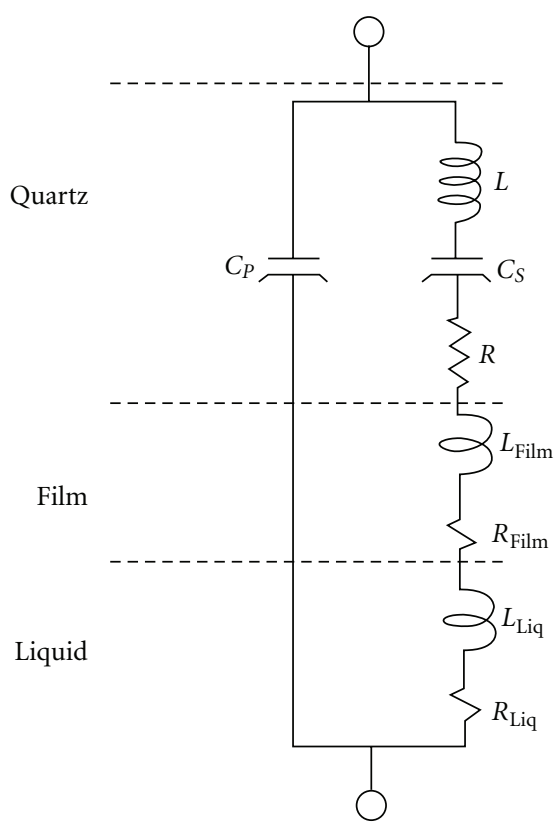

Figure 11: The Butterworth Van Dyke circuit can be modified, as shown, when the resonator is loaded with a film under a liquid.

transmission line can be reduced to a modified Butterworth Van Dyke circuit. Additional elements are added into the motional arm, as suggested in Figure 11. Here, the upper portion represents the unloaded quartz values. The portion below that represents the changes to the inductance and the resistance due to the mass loading of the film. Finally, the effects of the liquid are represented by the lower group of elements. For reasonably light film loadings, the film effects and the liquid effects can be treated as simple additive elements, as illustrated in Figure 11.

For the analysis of the transmission line model discussed above, the piezoelectric properties of the quartz were included. It is possible to take a complete solution for the resonator, including both the mechanical and electrical properties of the quartz as well as the loading of films and liquid without going to the transmission line equivalent. It is possible to solve for the behavior of the resonator under a variety of conditions. This approach is summarized in Figure 12 and described in [32].

At the top of the chart are indicated the parameters of the quartz, the film, and the liquid which are fed into a computational loop where the boundary conditions are satisfied. In order to satisfy these conditions, a quantity $Q_{4}$ is calculated in terms of some steady-state frequency $f$. $Q_{4}$ is defined as indicated by (3)

$$
J=-j \omega \varepsilon_{22} \frac{\Phi_{0}}{Q_{4}} .
$$

The details for the calculation of $Q_{4}$ are given in the appendix. The constant $Q_{4}$ defines the relation between the current density $J$ in amperes per sq meter and the voltage $\Phi_{0}$. From this relation, solutions under a variety of different conditions can be determined. It can be made specific to a

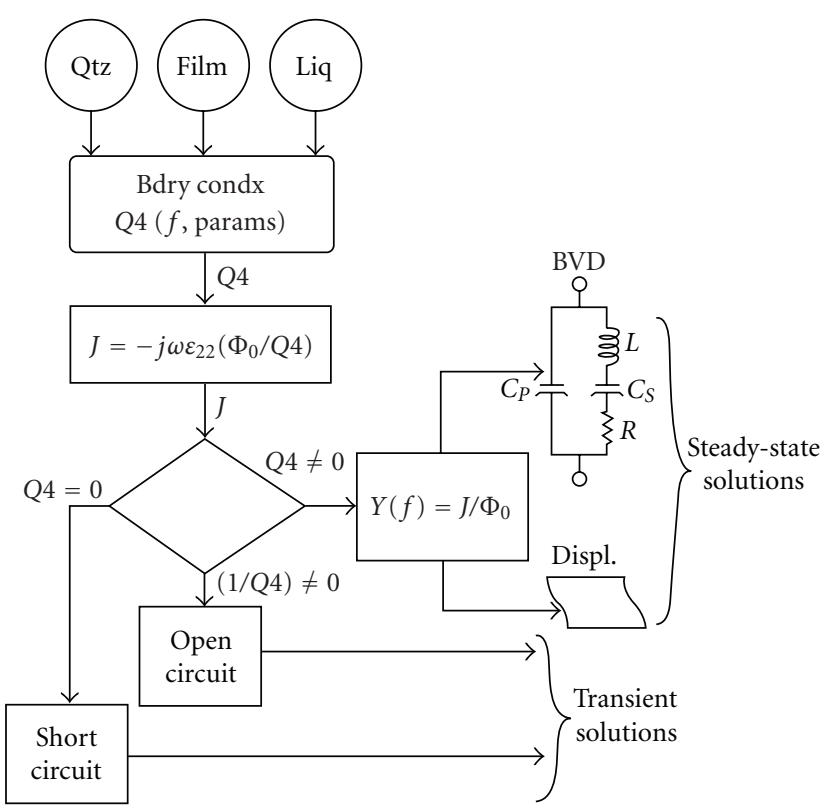

FIGURE 12: A flow diagram illustrating the complete electromechanical model.

crystal if desired by including a crystal area. In the case of the usual voltage source driven resonator, $\Phi_{0}$ is a constant.

The utility of the $Q_{4}$ concept is that the solutions for a variety of experimental conditions can be obtained from this single quantity. One can obtain steady-state solutions under constant voltage or constant current conditions as well as transient solutions under open circuit or short circuit conditions, as explained further in the following.

Since the frequency variation of $Q_{4}$ is known, and then one can easily calculate the current density as a function of frequency under the condition of constant $\Phi_{0}$. The admittance as a function of frequency is then simply given by the ratio $J /\left(\Phi_{0}\right)$. On the other hand, if one wanted to calculate the admittance under conditions of constant current, then in a similar manner, $\Phi_{0}$ can be calculated assuming a constant $J$. The admittance can be calculated again as the ratio of $J$ to $\Phi_{0}$.

Both of these cases are steady-state solutions where $Q_{4}$ is nonzero. As suggested on the right hand side of the figure, one can use $Q_{4}$ to obtain the magnitudes of the acoustic displacements in the resonant structure as shown under "Displ" in the figure. Alternatively, one can calculate the admittance spectrum and fit it to Lorentzian BVD elements. These two possibilities are the outputs of the steady-state solutions.

In the case of the transient solutions, we first consider the short-circuit case. In that case, the applied voltage is zero. From (1), we can see that there is a trivial solution where $J$ is zero. But there is another possibility of having a finite $J$ with a zero applied voltage. This occurs when $Q_{4}$ is zero. To satisfy the conditions in this case, the frequency must be taken to be complex. And the real part yields the resonant frequency while the imaginary part yields the decay time, related to the dissipation. Similarly, we can consider the case where the 


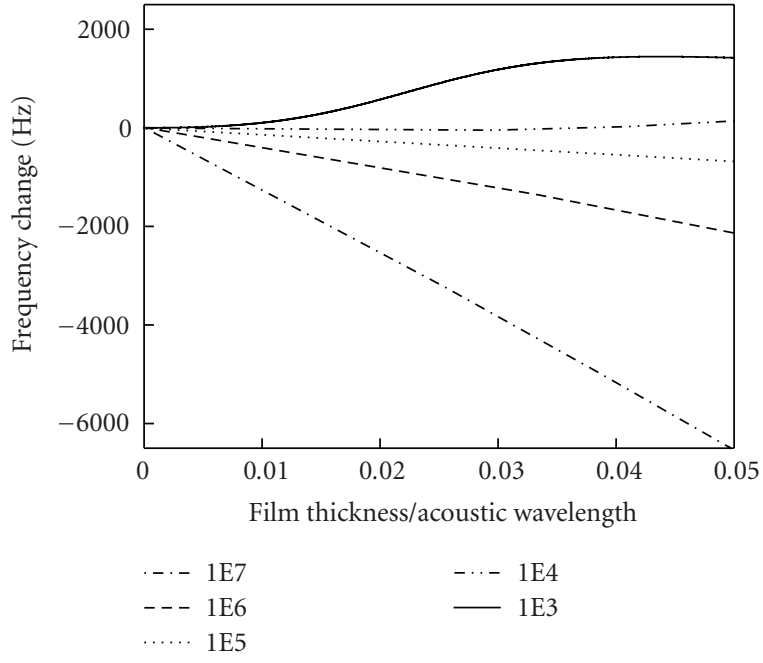

Figure 13: An example of the effect of the softness of a film under liquid on the frequency versus mass (thickness) relationship.

current is zero. Neglecting the trivial solution, we see that the voltage can be finite with a zero current if $1 / Q_{4}$ is zero. Again the frequency is complex and has the same interpretations as in the case of the short circuit.

We see that this electromechanical treatment can yield the full set of steady-state and transient solutions.

\section{Caveat}

Recently the special behavior of the resonance under load for the case of a film under liquid, when the film is very soft, having shear moduli less than $1 \times 10^{3} \mathrm{~Pa}$ has been discussed [33]. It is mentioned here because the behavior is so counterintuitive that the user may feel that the experimental results should be discounted. The type of behavior that can be observed is that the frequency-mass relation is nonlinear, and is not proportional to the Sauerbrey relation even for the thinnest films. It is even possible that the frequency increases with load. This behavior has been traced to an induced resonance in the soft film caused by the need to satisfy the film-liquid boundary conditions. An example of this is given in Figure 13.

In this figure, we have plotted the acoustic thickness along the abscissa instead of the actual film thickness. This was done so that the comparisons among the films with varying shear moduli are clear. The acoustic thicknesses are dependent upon the parameters of the film and are here expressed as a fraction of the wavelength of the acoustic wave in the film. The point here is to show the variations in the frequency-thickness behavior. The case for the stiffest film $\left(1 \times 10^{7} \mathrm{~Pa}\right)$ indicates Sauerbrey-type behavior. As the film softens with decreasing shear moduli, it is seen that slope of the curve decreases markedly and becomes almost flat for films of $1 \times 10^{4} \mathrm{~Pa}$. At the softest film value shown here $\left(1 \times 10^{3} \mathrm{~Pa}\right)$, the frequency can shift upward in a totally counterintuitive manner.

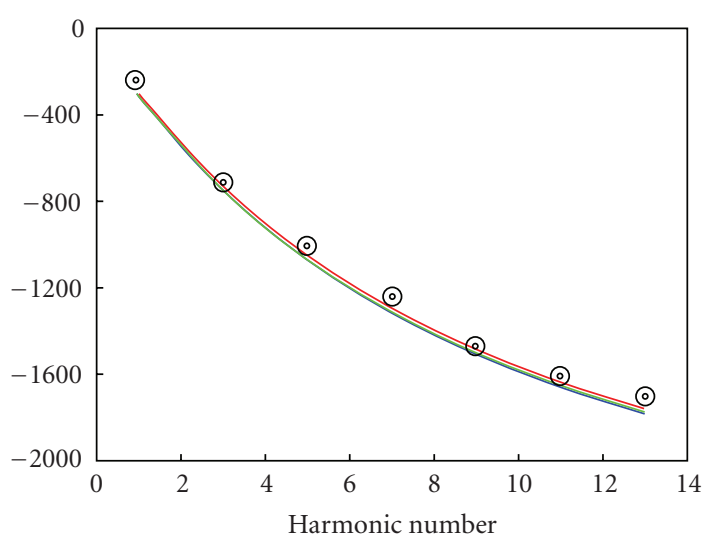

$\begin{array}{ll}\text { ○ Experiment } & - \text { Open Ckt } \\ \text { Voinova } & - \text { Electromech }\end{array}$

FIgURE 14: The behavior of the various models relative to the harmonic behavior of the resonances for a soft film of modulus $1.5 \times 10^{4} \mathrm{~Pa}$ is shown.

In order to compare the relative predictions of the various models, we plot in Figure 14 the results of the theoretical calculations using the three models: the Voigt-Voinova model, the open circuit model, and the full electromechanical treatment.

It is seen that the various models all yield virtually identical results. The models have been compared under other circumstances and they all yield virtually the same result. The user can use the model with which he is most comfortable with some assurance that it will yield results in agreement with other models.

To this point, we have tried to indicate the various QCM measurement methods and the various analytical methods. We have attempted to show the cautions which should be considered by the user. If considered, these cautions can lead to useful determinations of film properties with the QCM. In the remainder of this review, we shall highlight some of QCM applications related to biomimetic platforms and macromolecular interactions, primarily using the transient technique.

\section{QCM-D as a Sensor Platform for Biomacromolecular Interaction Studies}

With its ability to simultaneously detect mass and viscoelastic property changes, the quartz crystal microbalance with dissipation monitoring (QCM-D) is an ideal tool to study biological interactions [34-40]. Herein, we present four research topics that have had an impact in establishing QCM-D as a cutting-edge tool to study biomacromolecular interactions and provide a solid theoretical background to understand different modeling approaches. Lipid vesicles can interact with solid substrates in different ways depending on the surface properties [41]. First we introduce the fundamental sensor platform depending on surface-specific vesicle interactions. Then, we introduce an alpha helical 
(AH) peptide derived from the hepatitis $\mathrm{C}$ virus (HCV), which interacts with an intact lipid vesicle platform to cause a structural transformation, resulting in a complete bilayer $[35,37]$. We apply models to this system to examine how consideration of the adlayer's viscoelastic properties can improve data analysis [37]. In addition to these lipid-based platforms, other biological interactions such as protein adsorption can be studied with QCM-D [42]. We then illustrate the potential of the QCM-D technique in fundamental biological applications and compare it with more traditional biochemical assays by monitoring in situ AH peptide binding to lipid bilayers and cell membranes [34]. All of these studies have been conducted using crystals having a fundamental resonance frequency at $5 \mathrm{MHz}$.

5.1. Surface-Specific Vesicle Interactions. The early biomimetic systems focused on black lipid membranes [43], which were formed by painting a lipid bilayer across a gap in a solid surface. While many important studies came from this initial work, black lipid membranes' lack of robustness necessitated the development of improved systems. Characterization of black lipid membranes generally involves measuring the electrical resistance across the membrane by patch-clamping [44]. This characterization is nonspecific and many different lipid structures can indicate a strong electrical seal. Additionally, the lipid bilayer stability is very low because the structure is only supported at its edges and is essentially free-floating, making it more likely to rupture. Starting with Tamm and McConnell's work in 1985 [45, 46], solid-supported lipid bilayers (SLBs) became an alternative technique [47] with improved stability. The solid substrate to which the lipid bilayer is supported via hydrophilic interactions provides mechanical stability [41].

SLBs are a common model system to mimic biological membranes and are widely utilized as an experimental platform to study macromolecular interactions because their surface chemistry can be functionalized in a controlled fashion by changing the lipid composition or incorporating transmembrane proteins $[47,48]$. These are only examples of the multitude of functionalization possibilities. A promising strategy is to use bottom-up processes driven by molecular self-assembly, principles such as the formation of SLBs from vesicle solutions as shown in Figure 15. There are several common methods that are available to researchers to make solid-supported lipid bilayers. The two most common techniques are vesicle fusion $[47,48]$ and the LangmuirBlodgett (LB) or the Langmuir-Schaeffer (LS) transfer method [45]. After the pioneering work by Kasemo and coworkers $[39,41]$ using the quartz crystal microbalancedissipation (QCM-D) technique to study vesicle interactions with solid surfaces, vesicle fusion became a very popular experimental technique to form SLBs due to the ability of QCM-D to characterize the entire process, including the quality of the formed bilayer in terms of viscoelasticity and acoustic mass. It was demonstrated that vesicles adsorb irreversibly on $\mathrm{SiO}_{2}, \mathrm{Si}_{3} \mathrm{~N}_{4}, \mathrm{TiO}_{2}$, oxidized $\mathrm{Pt}$, oxidized $\mathrm{Au}$, and thiol-modified Au surfaces in a manner dependent on the surface's physical and chemical properties. Keller and
Kasemo [41] demonstrated for the first time that vesicles interact with different substrates to form three types of structures, namely, intact vesicle (Figures 15(b) and 15(d), lipid bilayer (Figures 15(a) and 15(c)), and lipid monolayer, based on the surface properties.

The QCM-D technique provided new insight into the vesicle fusion process on silicon oxide to form SLBs by revealing a two-step mechanism as shown in Figure 15(c). For the first time, a constructed SLB could be characterized in terms of its viscoelastic and mass properties. In addition, a significant benefit over previous characterization techniques including atomic force microscopy (AFM) and fluorescence recovery after photobleaching (FRAP) is that the entire SLB formation process-specifically the vesicle fusion process - can be easily monitored. $\Delta F_{\max }$ indicates the critical vesicle concentration at which vesiclevesicle and vesicle-surface interactions are optimized to induce vesicle rupture [39, 41]. Vesicle rupture creates bilayer islands with hydrophobic edges, which propagate further vesicle rupture until the lipid bilayer is complete and the edges are minimized. In terms of modeling, the SLBs thickness can be calculated by the Sauerbrey relationship [12] because the adsorbed layer is rigid as indicated by the relatively small dissipation changes shown in Figure 15(e). However, it should be noted that, at the critical vesicle concentration, the frequency and dissipation responses are overtone-dependent. Therefore, in order to better understand the structural transformation, it is necessary to use a viscoelastic model such as the VoigtVoinova model [14], which extends the QCM solutions to include viscoelastic effects. These thickness calculations, presented in Figures 15(e) and 15(f) were consistent over the frequency overtones with a film having a frequency-independent viscosity.

Whereas the critical vesicle concentration of adsorbed vesicles necessary to induce vesicle rupture can be thought of as a partial coverage of the surface with intact vesicles, vesicle adsorption on other substrates such as $\mathrm{TiO}_{2}$ and $\mathrm{Au}$ can form irreversible monolayers of intact vesicles, fully covering the surface as shown in Figures 15(d) and 15(f). Though these two systems are different in terms of surface coverage, the QCM-D frequency and dissipation responses are analytically similar. Both responses are overtone-dependent, necessitating the need for modeling that includes viscoelastic effects. In terms of forming an intact vesicle monolayer as a platform, there a number of parameters such as flow rate, vesicle size, and vesicle concentration that are critically important to platform reproducibility.

By using a viscoelastic model to calculate the adlayer thickness, it is possible to understand the degree of vesicle deformation. In Keller and Kasemo's work [41], they point out that adsorbed vesicles are no longer spherical but flatten out when adsorbed due to vesicle-surface and vesicle-vesicle interactions. They quantitatively analyzed this deformation process by calculating the theoretical frequency shift of an intact vesicle monolayer consisting of nondeformed vesicles $(107 \mathrm{~Hz})$ versus the experimental frequency shift $(90 \mathrm{~Hz})$ [41]. This work inspired researchers to revisit pathways to study vesicle fusion mechanisms and gave opportunities 
Solid-supported lipid bilayer

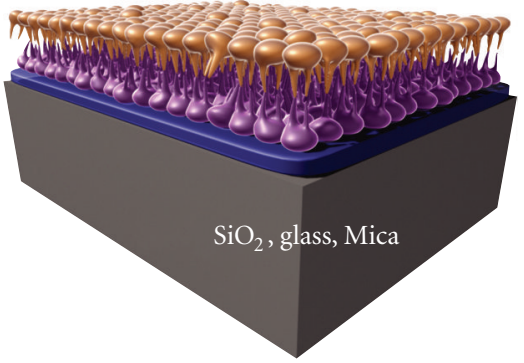

(a)

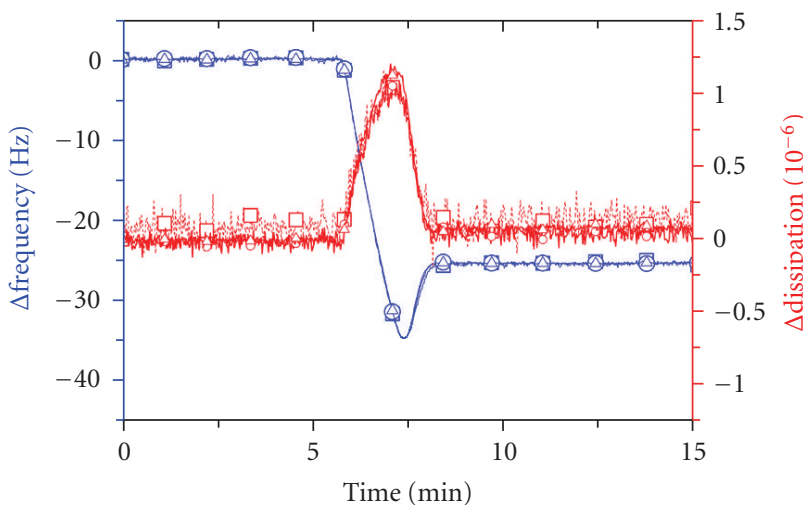

$-\square-3$ rd overtone

$-0-5$ th overtone

$-\triangleleft 7$ th overtone

(c)

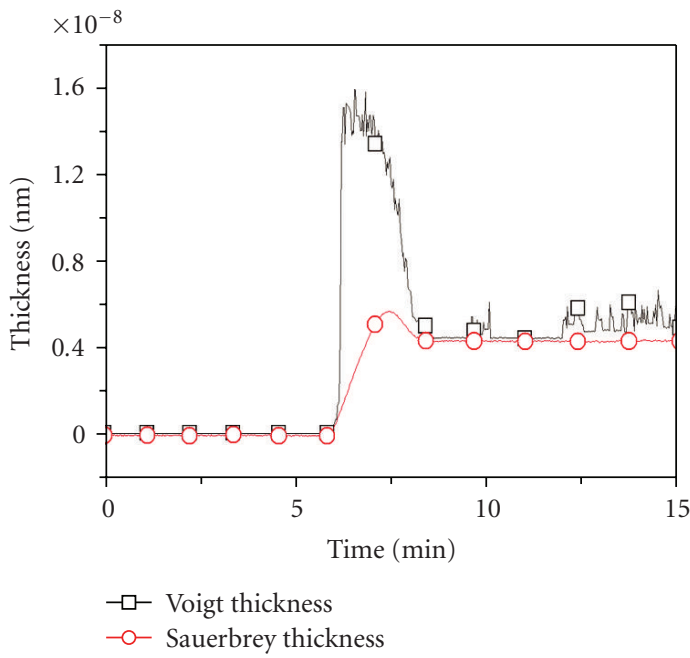

(e)

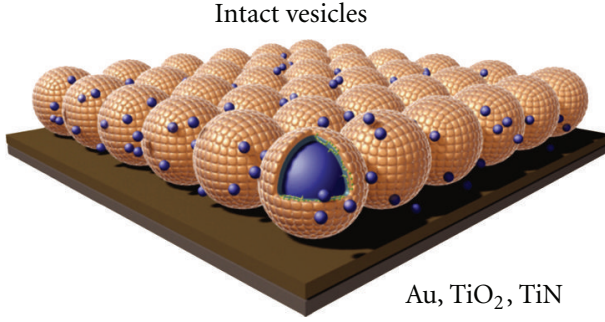

(b)

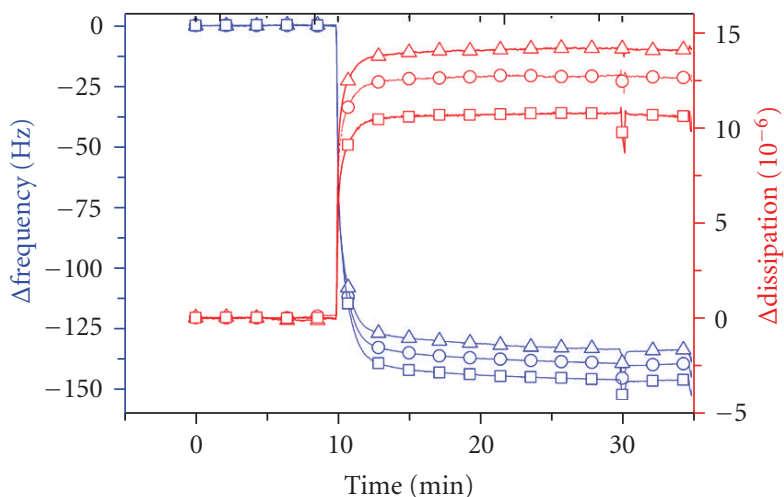

$-\square-3$ rd overtone

$-0-5$ th overtone

$\triangle-7$ th overtone

(d)

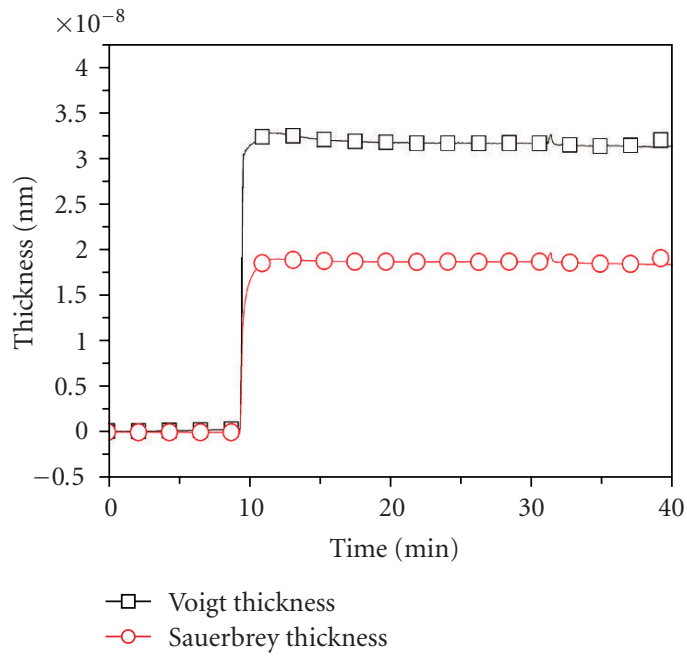

(f)

FIgURE 15: Surface specific vesicle adsorption kinetics on solid substrates. Illustration of (a) supported lipid bilayer on $\mathrm{SiO}_{2}$ and (b) intact vesicles on gold. (c) The typical two-step kinetics of vesicle fusion from small unilamellar vesicles to form a bilayer on $\mathrm{SiO}_{2}$. Note that the critical coverage occurred at 7.5 minutes. Three different overtones (3rd, 5th, and 7th) are also presented here for viscoelastic modeling. (d) The typical vesicle adsorption kinetics on a gold substrate are also presented. The huge mass uptake is caused by vesicle adorption. Due to the viscoelastic nature of vesicles, the overtones do not merge. (e) Comparison of two different thickness calculation-based Sauberbery and Voigt-based models to characterize the vesicle fusion process. Note the deviation at the critical coverage at 7.5 minutes due to the highly viscoelastic character of intact vesicles. (f) Comparison of two different thickness models by Sauerbrey and Voigt-based models for an adsorbed intact vesicle monolayer. Due to the viscoelastic nature of intact vesicles, the overtones do not merger. Since the adlayer's viscoelasticity causes high energy dissipation, the Sauerbrey model significantly underestimates the adlayer thickness compared with the Voigt-based model. 

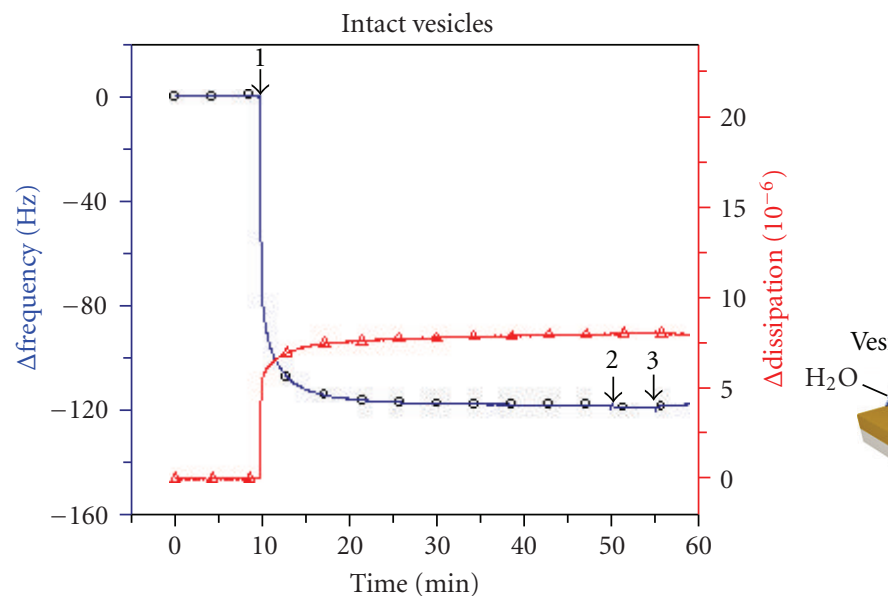

(a)
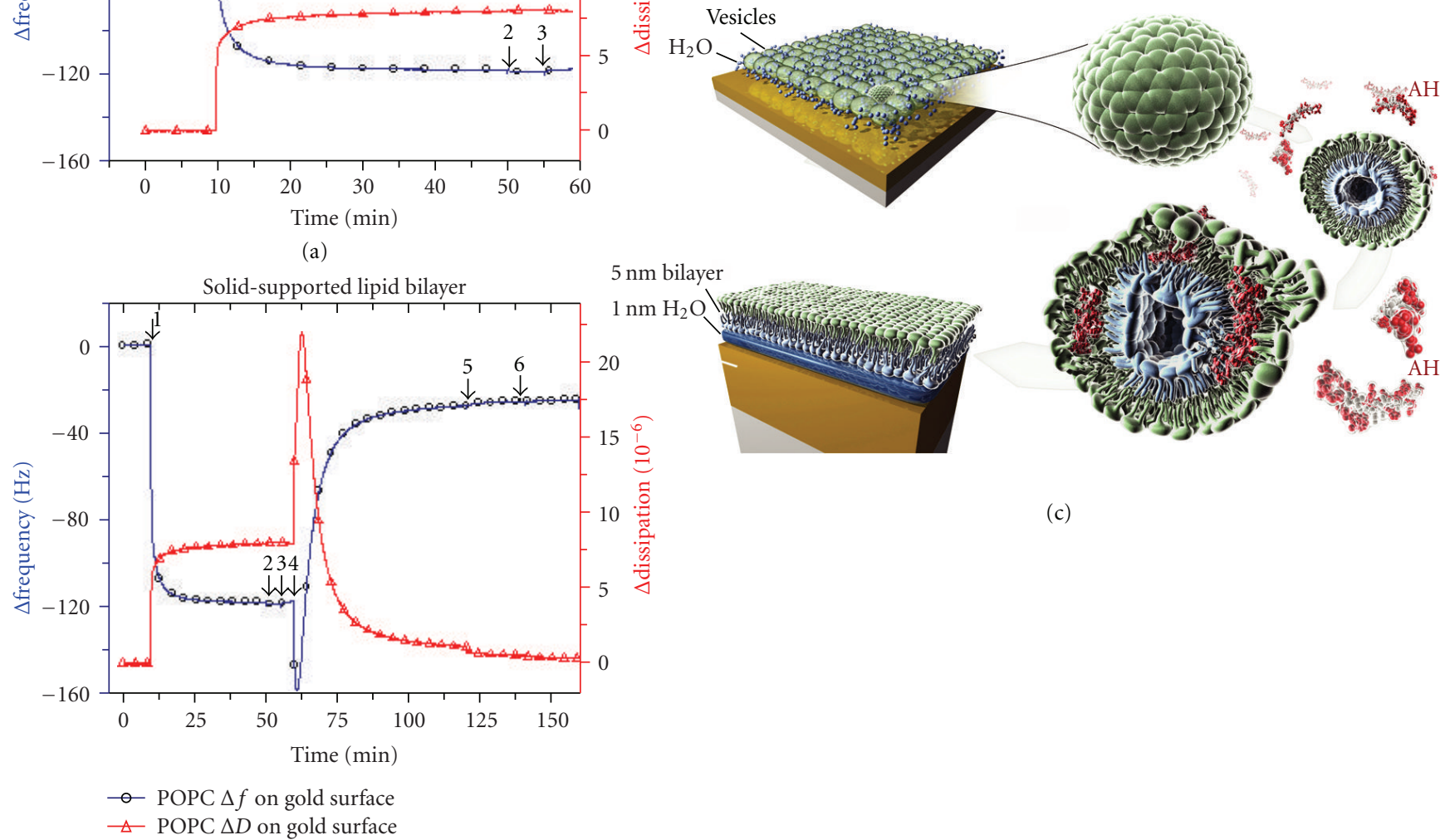

(c)

(b)

FIgURE 16: Structural transformation from intact vesicles to a lipid bilayer on a gold substrate mediated by an amphipathic $\alpha$-helix peptide $(\mathrm{AH})$ peptide. (a) Change in QCM resonant frequency and dissipation as a function of time for intact vesicles on oxidized gold. (B) Structural transformation from intact vesicles to a lipid bilayer $\Delta f(t)$ (blue curve) and $\Delta D(t)$ (red curve) show vesicle adsorption on oxidized gold. After 10 minutes (arrow 1) of stabilizing the frequency signal, a POPC vesicle solution $(0.1 \mathrm{mg} / \mathrm{mL}$, diameter $=59 \mathrm{~nm} \pm 0.2 \mathrm{~nm})$ was injected into the liquid cell. After 50 and 55 minutes (arrows 2 and 3), the same buffer was used for two washes and the stability of the intact vesicle adlayer on the gold surface was observed. (b) At 60 minutes (arrow 4), an AH peptide solution was added $(0.05 \mathrm{mg} / \mathrm{mL}$ ) to the intact vesicle adlayer on the gold surface. After 120 and 140 minutes (arrows 5 and 6), the same buffer was used for two washes and the stability of the bilayer formed on the gold surface was observed. (c) Illustration of vesicle rupture and fusion processes to form a supported bilayer on a gold substrate. Reprint from [35].

to study in more detail interfacial science study between biomacromolecules and substrates.

\subsection{Supported Bilayer Formed Peptide-Induced Vesicle Rup-} ture. Given the aforementioned experimental work done by Keller and Kasemo [41], one of the limitations of forming the bilayer platform via vesicle fusion is the need to have specific surface interactions, for example, hydrophilic interactions, and properties, for example, polarizability. In order to overcome this energetic problem, a new method was recently introduced to form a bilayer on different substrates such as $\mathrm{TiO}_{2}$ and $\mathrm{Au}$, known to have insufficient surface interactions and properties to rupture adsorbed vesicles, by introducing an amphipathic $\alpha$-helical $(\mathrm{AH})$ peptide as a vesicle destabilizing agent $[35,37]$. This process resulted in the formation of planar bilayers on $\mathrm{Au}$ and $\mathrm{TiO}_{2}$ surfaces, as seen in Figure 16.

By following the viscoelastic property and mass changes of this vesicle to bilayer transformation, we have successfully demonstrated that $\mathrm{AH}$ peptides destabilize and rupture the leaflets of intact lipid vesicles, allowing the ruptured vesicles to form planar bilayers [35, 37]. Regarding $\mathrm{AH}$ peptide's ability to destabilize vesicle structures, it is hypothesized that the peptide first creates instability on the vesicle surface by an electrostatic interaction. Based on similar biological systems 


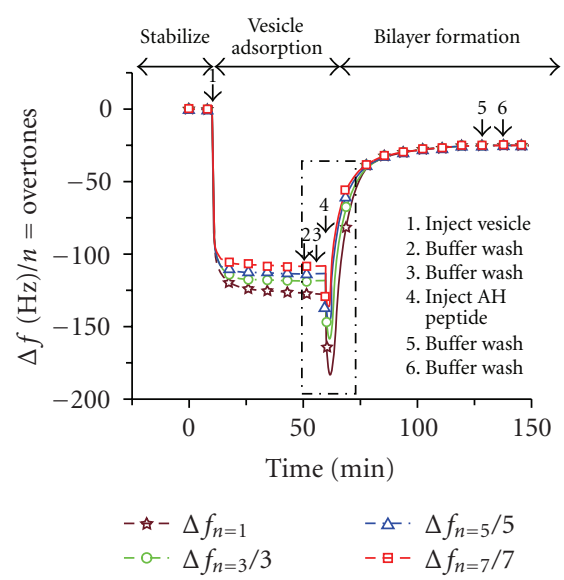

(a)

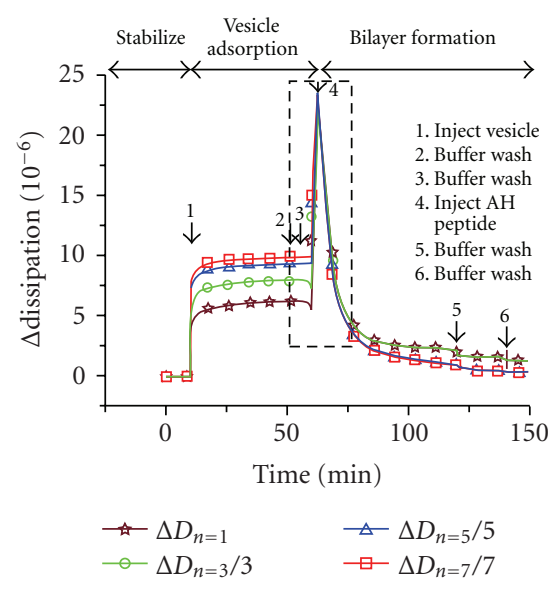

(d)

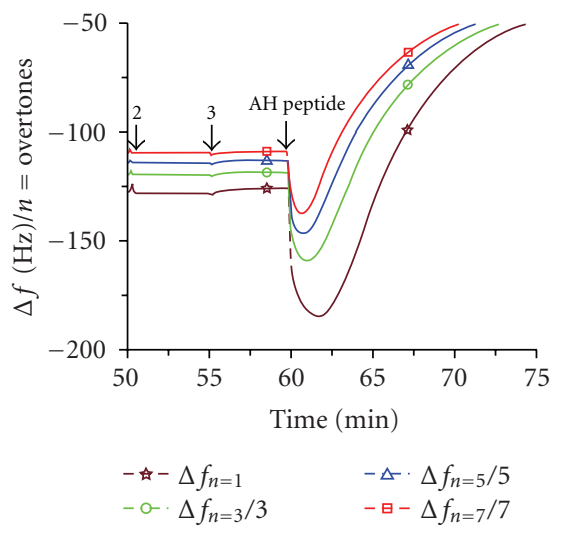

(b)

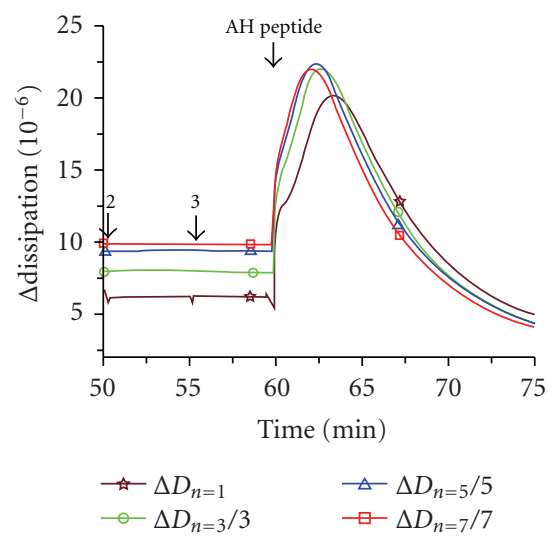

(e)
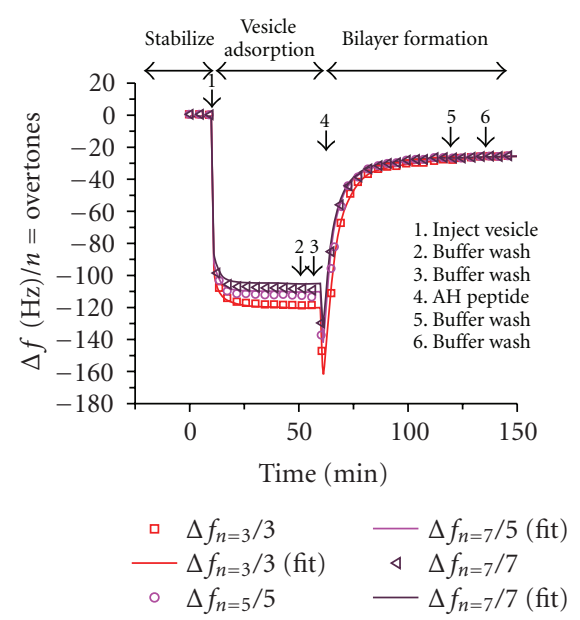

(c)

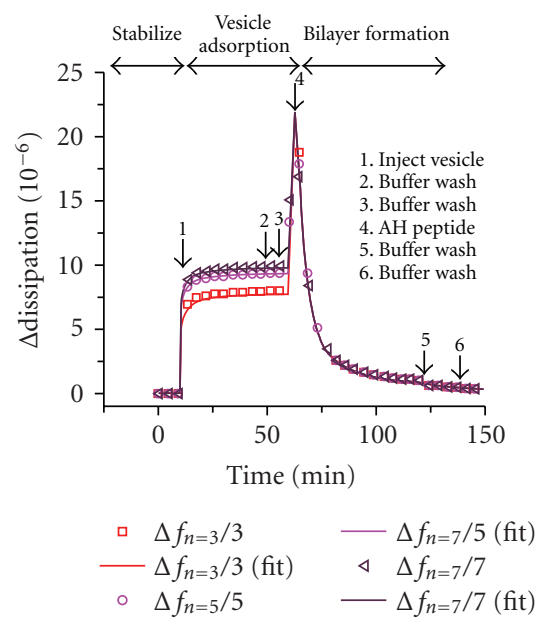

(f)

FIGURE 17: QCM-D signiatures for structural transformation from intact vesicles to a supported bilayer induced by AH peptide. (a) QCM-D adsorption kinetics of the first four overtones $\left(\Delta f_{n} / n\right.$ where $n=1,3,5,7$ vesicles in a Tris buffer solution $(150 \mathrm{mM} \mathrm{NaCl}, \mathrm{pH} 7.5)$ onto a gold surface. Vesicles are added after stabilizing the frequency signal for 10 minutes (see arrow 1). The film was then washed twice with the same buffer (see arrows 2 and 3). Upon addition of the AH peptide (see arrow 4), the vesicles rupture and form a bilayer on the gold surface. Two buffer washes were performed in order to ensure the stability of the film (see arrows 5 and 6). (b) Expanded view of (a). It is a detailed plot of $\mathrm{AH}$ peptide-induced vesicle rupture process from 50 to 75 minutes. The markers are also presented in order to distinguish the traces of the overtones (open star $\Delta f_{n}=1$, open circle $\Delta f_{n}=3$, open triangle $\Delta f_{n}=5$, and open square $\Delta f_{n}=7$, respectively. (c) Usie of a Voigt-based model to fit $\Delta f$ as shown in Figure 17(a) for the structural transformation from intact vesicles to a supported bilayer. The fit between the viscoelastic model ( $Q$-Tools with density $\rho=1100 \mathrm{~kg} / \mathrm{m} 3$ ) and the experimental data are presented. (d) Corresponding energy dissipation versus time plot demonstrates that the viscoelastic nature of the film changes during the structural transformation from soft vesicle layer to a rigid bilayer. (e) Expanded view of (c) to capture energy dissipation change corresponding to vesicle-AH peptide interaction. (f) Corresponding $\Delta D$ fit using same model. Reprint from [37].

studied in vivo, it is likely that this leads to expansion of the vesicles, causing a frequency decrease, as well as the creation of microvilli (finger-like structures) on the outer leaflet of the vesicles $[49,50]$. Vesicle expansion, resulting in a thicker, more viscoelastic film, and the formation of structures akin to microvilli on the vesicles' surfaces could explain the large increase in dissipation, which characterizes $\mathrm{AH}$ peptide's initial interaction with intact vesicles. Interestingly, $\mathrm{AH}$ peptide interaction with intact vesicles results in a final frequency shift relative to the initial state before vesicle adsorption of $25.5 \mathrm{~Hz} \pm 0.5$, and the final dissipation value is only $0.08 \times 10^{-6}$ (Figure 16(b)), both values corresponding to a complete rigid bilayer formed by the conventional vesicle fusion process. Figure 16(c) illustrated the vesicle rupture and fusion processes to form a supported lipid bilayer. Using the Sauerbrey relationship the resultant film thickness is in good agreement with that of a lipid bilayer, indicating a transition of the soft vesicle layer to a thin and rigid bilayer film as a result of the $\mathrm{AH}$ peptide action. With this strategy to form lipid bilayers on substrates, which do not permit vesicle fusion, researchers can take advantage of the electrical properties of $\mathrm{Au}$ and the biocompatibility of $\mathrm{TiO}_{2}$ to form 
new biomimetic structures than can be used in various applications such as biosensor and lab-on-a-chip devices. This novel process to form bilayers on $\mathrm{Au}$ and $\mathrm{TiO}_{2}$ shifts the focus on creating improved biomimetic systems away from materials-based problems.

Furthermore, in this model system as shown in Figure 16(b) is intriguing, since it demonstrated the "true" structural transformation from fully saturated single "intact vesicles" layer to lipid bilayer by foreign material, $\mathrm{AH}$ peptide. The structural properties can clearly be distinguished in situ by biochemical interactions between $\mathrm{AH}$ peptides and lipid molecules. After the "soft" intact vesicle layer is formed on the gold surface, the $\mathrm{AH}$ peptide only acts as "destabilizing agent" the layer of vesicles that opt to rupture due to interaction between vesicle-vesicle, and vesicle-surface (e,g., in this particular case, polarization of gold surface and hydrophilic interaction with $\mathrm{TiO}_{2}$ ).

Unlike the classical vesicle rupture mechanism on an $\mathrm{SiO}_{2}$ surface, this system made it possible to capture the structural transformation leading to the formation of a "rigid" bilayer on any solid substrate. To analyze this transformation, Cho et al. [37] first examined the validity of the Sauerbrey relation for both the "soft" and "rigid" layers by calculating adsorbed film thickness. While the Sauerbrey equation is still popular and simple to use for estimating film thickness, more accurate models have been derived to describe the effects of adding various types of foreign masses on a quartz crystal. In order to better understand the structural transformation from a "soft" to a "rigid" film, Cho et al. [37] compared the observed changes with calculations from the Voigt-Voinova model, which extends the QCM solutions beyond the Sauerbrey analogies to include viscoelastic effects. These changes were remarkably consistent over the harmonic frequencies with a film having a frequency independent viscosity. The mechanical loss tangent which is proportional to the ratio of the viscosity to the shear modulus is a parameter useful in characterizing a film as either solid-like or liquid-like. A comparison of the mechanical loss tangent as determined by the Voigt-Voinova fit using a frequency independent viscosity shows that the final "soft" vesicle layer had a $\tan \delta$ greater than unity, while that of the "rigid" bilayer had a tan delta less than unity. This agrees with the notion that the "soft" layer is more liquidlike, while the "rigid" layer is solid-like.

5.3. Protein Adsorption. Numerous QCM-D studies have focused on small biomolecules such as proteins. These studies have included protein-protein and protein-surface interactions as well as conformational changes in protein films [51-60]. Here we describe a particular 2001 study by Höök et al. [42]. It is notable for characterizing the structural transformation of a mussel adhesive protein adsorbed onto a hydrophobic methyl-terminated surface. It is an ideal example to demonstrate the usefulness of simultaneous detection of mass and viscoelastic property changes to characterize a dynamic, multistep biological process. The adsorption kinetics of the protein and subsequent structural transformation induced by cross-linking are monitored in terms of frequency and dissipation changes in order to understand the film properties.

As seen in Figure 18, the initial step involves adsorption of the protein onto a hydrophobic substrate. There is a large dissipation change $\left(D_{1}=15.8 \pm 0.2\right)$ associated with the binding process, which indicates that the resulting protein film is highly viscoelastic. The normalized frequency and dissipation overtone deviations (25\% for $n=3$ and $34 \%$ for $n=5$ ) are a result of the energy damping caused by the soft film properties. After adding a cross-linking agent, the dissipation decreases to nearly zero, indicating the formation of a nonviscoelastic film. By analyzing this change in dissipation upon cross-linking, it is clear that there is a structural transformation of the protein film on a macroscopic level because there is a significant change in the film's physical properties.

In terms of modeling, this system is very similar to the $\mathrm{AH}$ peptide-induced vesicle rupture process because both involve the structural transformation of a non-Sauerbrey regime to a Sauerbrey regime. Initially, the protein film has a high bound water content and requires a viscoelastic model to accurately characterize this non-Sauerbrey regime. Following cross-linking, the protein film becomes rigid and the associated mass decrease is predicted to be caused by a decease in bound water content, which is in agreement with the dissipation data. A lower bound solvent content characterizes more rigid films and therefore they satisfy all the conditions necessary to use the Sauerbrey relationship.

A very interesting aspect of this study is that the entire protein adsorption and cross-linking events were repeated in $\mathrm{D}_{2} \mathrm{O}$ in order to check the validity of the fitting parameters that satisfy the Voigt model. This experimental step confirmed the validity of the Voigt model to describe the protein film in both the Sauerbrey and non-Sauerbrey regimes as well during the structural transformation.

\subsection{Binding Dynamics of AH Peptide to Artificial Cell Mem-} branes. As increasing advances in bionanotechnology are made, there is an increasing need to engineer biomembrane platforms, which use phospholipid bilayers to support, protect, and organize membrane proteins. New lipid-based platforms have enabled a wide array of new research on biological membranes by (a) controlling the parameters of the materials, such that optimal platforms are ensured for promoting the interaction with target molecules, (b) studying the total saturation of the acyl chains, and the interaction of the membrane lipids with the target since lipid phase behavior is influenced by the lipid composition, (c) evaluating the effect of changing temperature and $\mathrm{pH}$ conditions, which are known to alter the biophysical properties of lipids, and (d) adding accessory biologic factors, such as an insoluble detergent complex (raft) that can be utilized to enhance the interaction with the target. Most researchers apply a "bottom-up" strategy to achieve this goal by modifying phospholipid compositions and incorporation transmembrane protein into the bilayer system. Under normal conditions, transmembrane conformation and proper protein attachment to membrane are necessary 

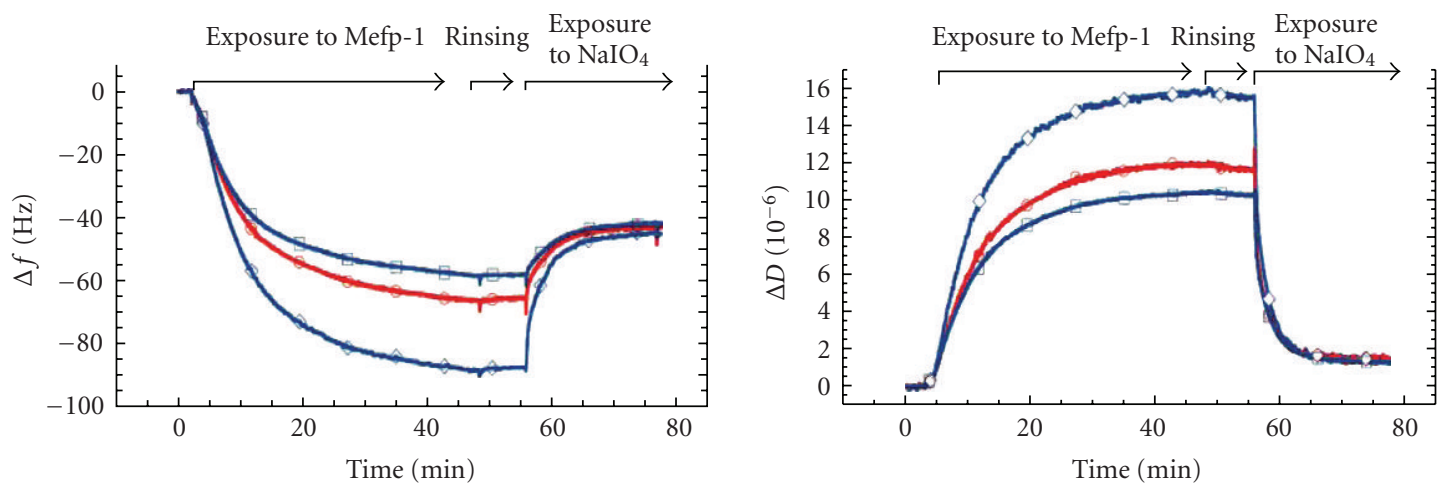

$$
\begin{aligned}
& \smile \Delta f_{n=1} \\
& \smile \Delta f_{n=3} / 3 \\
& \square \Delta f_{n=5} / 5
\end{aligned}
$$

(a)

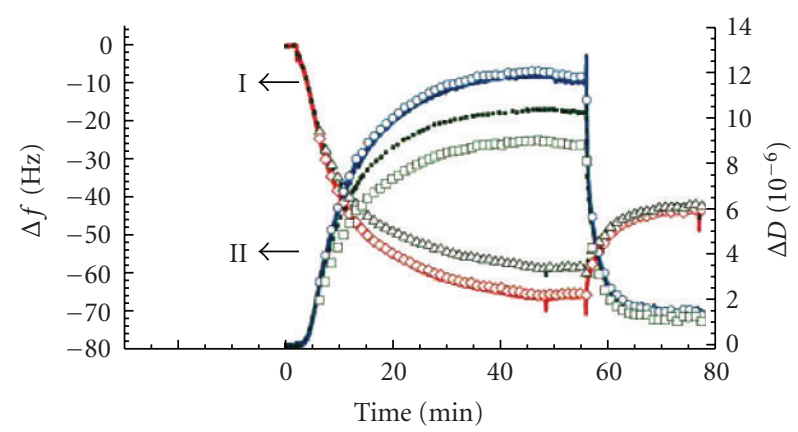

$$
\begin{aligned}
& \frac{\mathrm{I}}{\cdots \cdot \Delta f_{n=3} / 3} \\
& \diamond \Delta f_{n=3} / 3 \text { (fit) } \\
& \Delta \Delta f_{n=5} / 5 \text { (fit) } \\
& \text { II } \Delta D_{n=3} / 3 \\
& \text { … } \Delta D_{n=5} / 5 \\
& \text { - } \Delta D_{n=3} / 3 \text { (fit) } \\
& \text { ㅁ } \Delta D_{n=5} / 5 \text { (fit) }
\end{aligned}
$$

(c)

$$
\begin{aligned}
& \prec \Delta D_{n=1} \\
& \smile \Delta D_{n=3} / 3 \\
& \square \Delta D_{n=5} / 5
\end{aligned}
$$

(b)

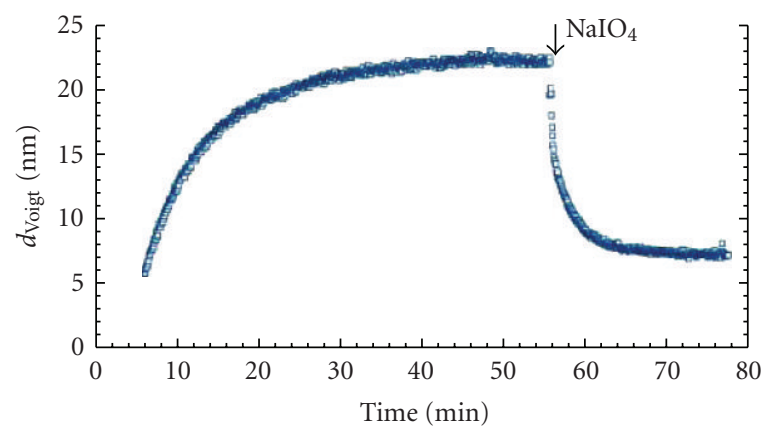

FIGURE 18: QCM-D response of overtones as a function of time for protein adsorption and subsequent cross-linking. (a) Exposure of the methyl-terminated surface to a buffer solution containing $25 \mathrm{mg} / \mathrm{mL}$ Mefp- 1 in $0.1 \mathrm{M}$ acetate buffer $(0.75 \mathrm{M} \mathrm{NaCl}$, pH 5.5$)$, followed by exchange of the protein solution for a pure buffer solution. The cross-linking behavior of Mefp-1 was subsequently followed by addition of the same buffer solution containing $1 \mathrm{mM} \mathrm{NaIO}_{4}$. (b) Corresponding dissipation as a function of time for experiment shown in (a). (c) The best fit between the viscoelastic model (obtained using Q-Soft software(Q-Sense AB)), and the experimental data shown in Figure 17(a). (d) Change in the effective thickness with respect to time obtained from the fit shown in (c). Reprint from [42].

via complex electrostatic interactions between proteins and phospholipids were necessary for proper functions. In here, as sensor system, we discussed the new "membrane on a chip" system employing "top-to-bottom" approach [47, 48], namely, utilizing cell derived the microsome to form functional membrane to study protein-protein interaction. As an model system, we presented the examples for monitoring the association of the NS5A AH peptide with a model lipid bilayer and cell-derived membranes. Furthermore, compare these results to conventional biochemical floatation assay to check the validity of new platform formed by "top-down" approach [34].

Conventional biological responses are often triggered by functional protein receptors. In order to capture this response, Cho et al. [34] made use of a novel "membrane-ona-chip" system wherein the binding dynamics of a synthetic peptide corresponds to the NS5A AH to lipid bilayers and cell-derived membrane by "top-down" approach that could be studied in real time using the quartz crystal microbalance with dissipation (QCM-D) method. Moreover, they found that significantly more peptide bound when cellular-derived membranes in which accompany with protein receptors. The direct evidence protein-peptide interaction was captured by eliminating protein receptors by prior treatment of the cellular membranes with trypsin. A control peptide, whose interaction with membranes involves trypsin-insensitive glycosaminoglycans, was not altered by the protease treatment. Similar results were obtained using standard biochemical membrane flotation assays of NS5A $\mathrm{AH}$-containing proteins that support the validity of QCM-D platform used as bioassay. The aforementioned experimental results demonstrated the potential of QCM-D for studying these types of protein-membrane interactions as well as a broad range of problems involving membrane proteins 


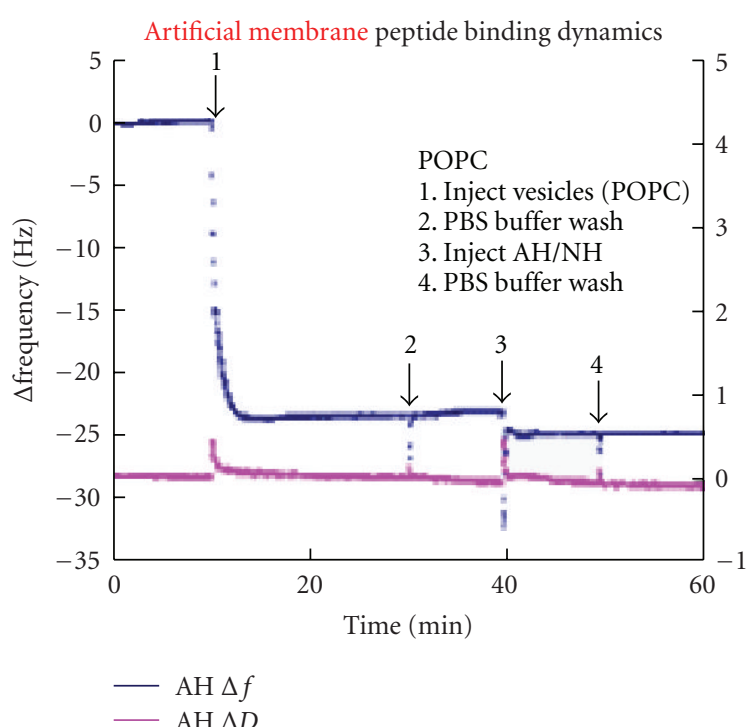

(a)

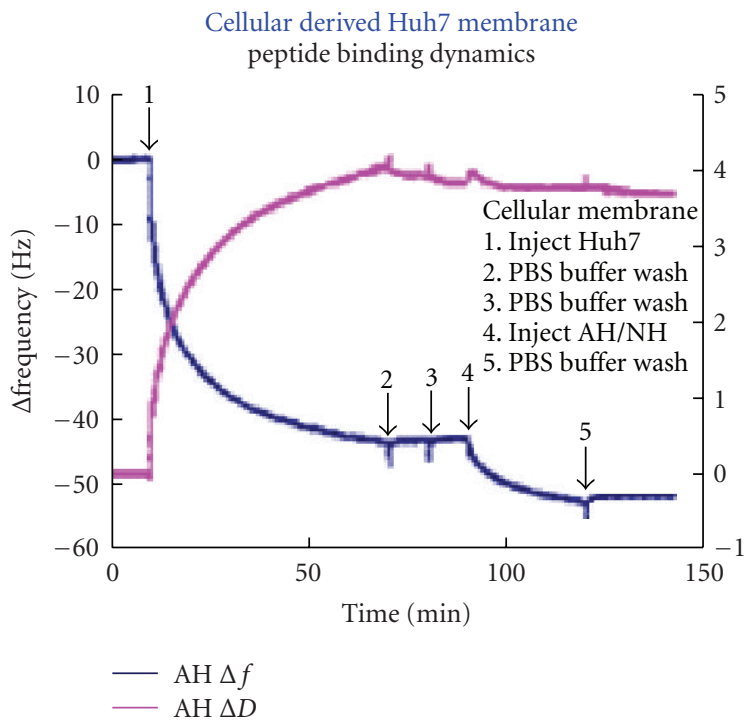

(c)

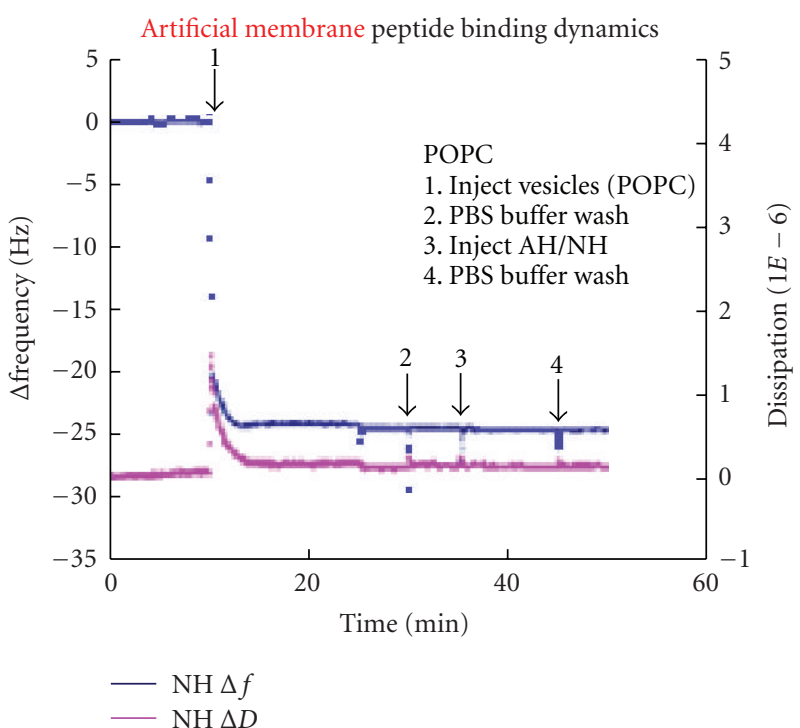

(b)

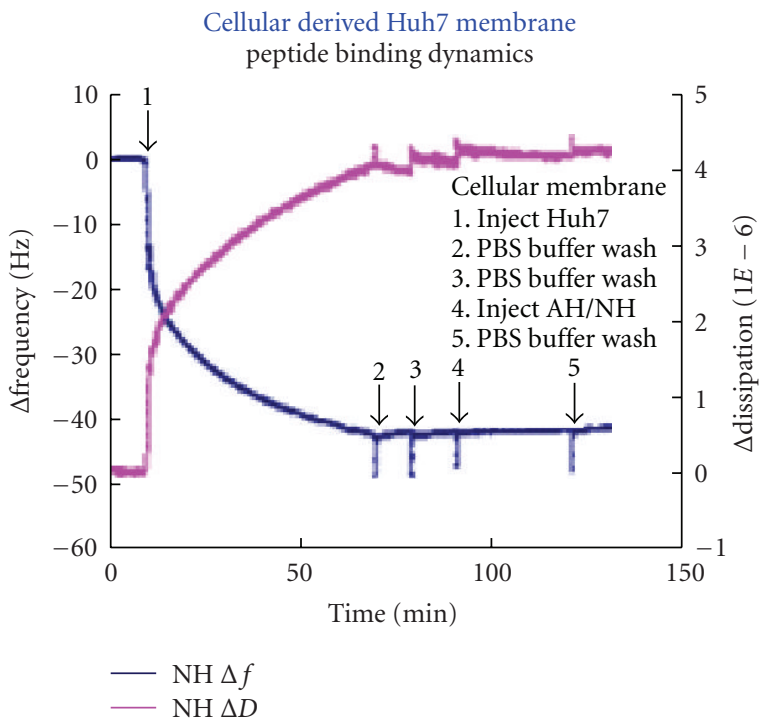

(d)

FIGURE 19: QCM-D analysis of AH peptide binding to a quartz crystal coated with POPC model lipid membrane and a cell-derived membrane. (a) AH peptide binding dynamics on a POPC model lipid bilayer. Frequency, $\Delta f(t)$, and dissipation, $\Delta D(t)$, changes detected by QCM-D as a function of time were recorded. (b) NH peptide binding dynamics to POPC model lipid bilayers. NH contains three mutations designed to disrupt the hydrophobic face of $\mathrm{AH}$. (c) Binding of $\mathrm{AH}$ peptide to Huh7-derived microsome coated onto an $\mathrm{SiO}_{2}$ quartz crystal substrate. The data show that the microsomes saturated at a higher mass than the model POPC membrane, presumably due to the presence of proteinaceous components. They also show by the dissipation value that those proteinaceous components are associated with higher viscoelastic energy dissipation, as expected. (d) No binding of $\mathrm{NH}$ peptide to Huh7-derived membranes adsorbed on an $\mathrm{SiO}_{2}$ surface. $\mathrm{Note}$ that there are no changes in either the frequency or dissipation, which suggests that there is no binding to Huh7-derived membranes. Reprint from [34].

or lipids. Furthermore, using "top-down" approach with conventional bioseparation techniques will provide powerful tool to create functional platform to study interaction and biomacromolecular dynamics. The main advantage of the potential biosensor described in this study using QCM-D technique is that it is simple and quick to use and that it shows the real-time kinetics of the interactions happening on the lipid bilayer derived from the cells that are comparable to the results obtained by traditional biochemical analysis. Standard biological assays can take days to complete experiments. However, using the unique "lab on a chip" approach of QCM-D can reduce assay time to hours. 


\section{Conclusions}

It is hoped to have provided a view of the use of the quartz crystal microbalance as a sensor to probe various aspects of biomacromolecular assemblies. The importance of both the frequency change and dissipation change during the assembly process has been emphasized as well as the various means for exploring the source of these changes through modeling. The special information that can be gained using the QCM as one of the tools in the arsenal of detectors for macromolecular processes is illustrated by several examples. Among these are the study of surface specific interactions on lipid vesicles, the demonstration of a vesicle cell rupture caused by the adsorption of a peptide onto its surface, and the use of model fits to these processes. The importance of capturing both the frequency and dissipation data is demonstrated.

\section{Appendix}

We have gathered here the specific relations detailing the calculation of the quantity $Q_{4}$. This has been made available to the reader who might be interested in doing the quantitative computations:

- $e_{26}$ is the appropriate piezo constant for quartz having the value $-0.095 \mathrm{C} \mathrm{m}^{-1}$

- $\varepsilon_{22}$ is the appropriate dielectric constant for the quartz with the value $3.982 \times 10^{-11} \mathrm{fd} / \mathrm{m}$;

- $c_{66}$ is the appropriate shear modulus for the quartz with the value $2.901 \times 10^{10} \mathrm{~N} \mathrm{~m}^{-2}$

- $d_{\mathrm{Q}}$ is the quartz thickness in $\mathrm{m}$,

- $\rho_{\mathrm{Q}}$ is the quartz density having the value of $2649 \mathrm{kgm}$ $\mathrm{m}^{-3}$;

- $\tilde{c}_{66}$ is the stiffened quartz shear modulus;

- $k_{Q}$ is the acoustic wave propagation constant in the quartz;

- $Q_{1}$ is a collection of terms for the quartz;

- $Q_{2}$ is another set of terms for the quartz;

- $\rho_{L}$ is the density of the liquid;

- $\eta_{L}$ is the viscosity of the assumed Newtonian liquid;

- $\rho_{F}$ is the density of the film on the quartz;

- $\mu_{F}$ is the shear modulus for the film in $\mathrm{N} \mathrm{m}^{-2}$

- $k_{F}$ is the acoustic wave propagation constant in the film,

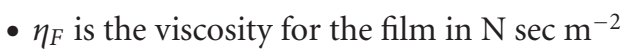

- $k_{L}$ is the acoustic wave propagation constant in the liquid;

- $T_{1}$ is a collection of terms involving the film and the liquid;

- $T_{2}$ is a second collection of terms involving the film and the liquid;

- $Q_{3}$ is another collection of terms;

$$
\begin{aligned}
& \tilde{c}_{66}=c_{66}+\frac{e_{26}^{2}}{\varepsilon_{22}}, \\
& k_{Q}=\omega \sqrt{\frac{\rho_{Q}}{\widetilde{c}_{66}}}, \\
& Q_{1}=j k_{Q} \tilde{c}_{66}\left(1-e^{-j k_{Q} d_{Q}}\right), \\
& Q_{2}=j k_{Q} \tilde{c}_{66}\left(1-e^{\left.j k_{Q} d_{Q}\right)},\right. \\
& \tilde{\mu}_{F}=\mu_{F}+j \omega \eta_{F}, \\
& k_{F}=\omega \sqrt{\frac{\rho_{F}}{\tilde{\mu}_{F}}}, \\
& k_{L}=\omega \sqrt{\frac{\rho_{L}}{j \omega \eta_{L}}}, \\
& T_{1}=1+\frac{j k_{F} \tilde{\mu}_{F}-\omega k_{L} \eta_{L}}{j k_{F} \tilde{\mu}_{F}+\omega k_{L} \eta_{L}} e^{j 2 k_{F} d_{F},}, \\
& T_{2}=1-\frac{j k_{F} \tilde{\mu}_{F}-\omega k_{L} \eta_{L}}{j k_{F} \tilde{\mu}_{F}+\omega k_{L} \eta_{L}} e^{j 2 k_{F} d_{F}}, \\
& Q_{3}=j k_{F} \tilde{\mu}_{F} \frac{T_{2}}{T_{1}},
\end{aligned}
$$

We finally obtain the expression to be used for the quantity $Q_{4}$,

$$
\begin{aligned}
Q_{4}= & \left\{\frac{e_{26}^{2}}{\varepsilon_{22}}\right. \\
& \times \frac{\left(1-e^{-j k_{Q} d_{Q}}\right)+\left(1-e^{j k_{Q} d_{Q}}\right)\left(\left(Q_{1}-Q_{3}\right) /\left(Q_{2}+Q_{3}\right)\right)}{j k_{Q} \tilde{c}_{66}\left(e^{-j k_{Q} d_{Q}}-e^{j k_{Q} d_{Q}}\left[\left(Q_{1}-Q_{3}\right) /\left(Q_{2}+Q_{3}\right)\right]\right)} \\
& \left.-d_{Q}\right\} .
\end{aligned}
$$

\section{Acknowledgment}

N.J. Cho is a recipient of an Americal Liver Foundation Postdoctoral Fellowship Award and a Stanford Dean's Fellowship. 


\section{References}

[1] A. Arnau Vives, Piezoelectric Transducers and Applications, vol. 26, Springer, Berlin, Germany, 2008, 125 illustration.

[2] C. Lu and A. W. Czanderna, Applications of Piezoelectric Quartz Crystal Microbalances, Elsevier, New York, NY, USA, 1984.

[3] D. A. Buttry and M. D. Ward, "Measurement of interfacial processes at electrode surfaces with the electrochemical quartz crystal microbalance," Chemical Reviews, vol. 92, no. 6, pp. 1355-1379, 1992.

[4] V. E. Bottom, Introduction to Quartz Crystal Unit Design, Van Nostrand Reinhold, New York, NY, USA, 1982.

[5] R. W. Cernosek, S. J. Martin, A. R. Hillman, and H. L. Bandey, "Comparison of lumped-element and transmissionline models for thickness-shear-mode quartz resonator sensors," IEEE Transactions on Ultrasonics, Ferroelectrics, and Frequency Control, vol. 45, no. 5, pp. 1399-1407, 1998.

[6] H. Muramatsu, E. Tamiya, and I. Karube, "Computation of equivalent circuit parameters of quartz crystals in contact with liquids and study of liquid properties," Analytical Chemistry, vol. 60, no. 19, pp. 2142-2146, 1988.

[7] S. J. Martin, V. E. Granstaff, and G. C. Frye, "Characterization of a quartz crystal microbalance with simultaneous mass and liquid loading," Analytical Chemistry, vol. 63, no. 20, pp. 22722281, 1991.

[8] H. L. Bandey, A. R. Hillman, M. J. Brown, and S. J. Martin, "Viscoelastic characterization of electroactive polymer films at the electrode/solution interface," Faraday Discussions, vol. 107, pp. 105-121, 1997.

[9] A. Arnau, "A review of interface electronic systems for AT-cut quartz crystal microbalance applications in liquids," Sensors, vol. 8, no. 1, pp. 370-411, 2008.

[10] H. L. Bandey, S. J. Martin, R. W. Cernosek, and A. R. Hillman, "Modeling the responses of thickness-shear mode resonators under various loading conditions," Analytical Chemistry, vol. 71, no. 11, pp. 2205-2214, 1999.

[11] B. D. Vogt, E. K. Lin, W.-I. Wu, and C. C. White, "Effect of film thickness on the validity of the sauerbrey equation for hydrated polyelectrolyte films," Journal of Physical Chemistry $B$, vol. 108, no. 34, pp. 12685-12690, 2004.

[12] G. Sauerbrey, "Verwendung von Schwingquarzen zur Waegun duenner Schichten und zur Mikrowaegung," Zeitschrift für Physik, vol. 155, p. 206, 1959.

[13] M. Rodahl, F. Hook, C. Fredriksson, et al., "Simultaneous frequency and dissipation factor QCM measurements of biomolecular adsorption and cell adhesion," Faraday Discussions, vol. 107, pp. 229-246, 1997.

[14] M. V. Voinova, M. Jonson, and B. Kasemo, "'Missing mass' effect in biosensor's QCM applications," Biosensors and Bioelectronics, vol. 17, no. 10, pp. 835-841, 2002.

[15] M. Ferrari, V. Ferrari, and K. Kanazawa, "Dual-harmonic oscillator for quartz crystal resonator sensors," Sensors and Actuators A, vol. 145-146, no. 1-2, pp. 131-138, 2008.

[16] J. R. Vig, "Dual-mode oscillators for clocks and sensors," in Proceedings of the IEEE Ultrasonics Symposium (ULTSYM '99), vol. 2, pp. 859-868, Caesars Tahoe, Nev, USA, October 1999.

[17] A. L. Kipling and M. Thompson, "Network analysis method applied to liquid-phase acoustic wave sensors," Analytical Chemistry, vol. 62, no. 14, pp. 1514-1519, 1990.

[18] R. Lucklum, C. Behling, R. W. Cernosek, and S. J. Martin, "Determination of complex shear modulus with thickness shear mode resonators," Journal of Physics D, vol. 30, no. 3, pp. 346-356, 1997.
[19] S. M. Yoon, N.-J. Cho, K. Kanazawa, and C. W. Frank, "Analyzing spur-distorted impedance spectra for the QCM," submitted to Journal of Sensors.

[20] M. Rodahl, F. Hook, and B. Kasemo, "QCM operation in liquids: an explanation of measured variations in frequency and Q factor with liquid conductivity," Analytical Chemistry, vol. 68, no. 13, pp. 2219-2227, 1996.

[21] P. J. Cumpson and M. P. Seah, "The quartz crystal microbalance; radial/polar dependence of mass sensitivity both on and off the electrodes," Measurement Science \& Technology, vol. 1, no. 7, pp. 544-555, 1990.

[22] L. Wimmer, S. Hertl, J. Hemetsberger, and E. Benes, "New method of measuring vibration amplitudes of quartz crystals," Review of Scientific Instruments, vol. 55, no. 4, pp. 605-609, 1984.

[23] M. A. Cooper, "Biosensing using rupture event scanning (REVS)(TM)," Measurement Science \& Technology, vol. 14, no. 11, pp. 1888-1893, 2003.

[24] M. Edvardsson, M. Rodahl, and F. Hook, "Investigation of binding event perturbations caused by elevated QCM-D oscillation amplitude," Analyst, vol. 131, no. 7, pp. 822-828, 2006.

[25] J. G. Miller and D. I. Bolef, "Acoustic wave analysis of the operation of quartz-crystal film-thickness monitors," Journal of Applied Physics, vol. 39, no. 12, pp. 5815-5816, 1968.

[26] C.-S. Lu and O. Lewis, "Investigation of film-thickness determination by oscillating quartz resonators with large mass load," Journal of Applied Physics, vol. 43, no. 11, pp. 4385-4390, 1972.

[27] T. Nomura and A. Minemura, "Behavior of a piezoelectric quartz crystal in an aqueous-solution and the application to the determination of minute amount of cyanide," Nippon Kagaku Kaishi, pp. 1621-1625, 1980.

[28] A. Bund, A. Baba, S. Berg, et al., "Combining surface plasmon resonance and quartz crystal microbalance for the in situ investigation of the electropolymerization and doping/dedoping of poly(pyrrole)," Journal of Physical Chemistry $B$, vol. 107, no. 28, pp. 6743-6747, 2003.

[29] C. Behling, R. Lucklum, and P. Hauptmann, "The nongravimetric quartz crystal resonator response and its application for determination of polymer shear modulus," Measurement Science \& Technology, vol. 9, no. 11, pp. 1886-1893, 1998.

[30] M. Rodahl and B. Kasemo, "On the measurement of thin liquid overlayers with the quartz-crystal microbalance," Sensors and Actuators A, vol. 54, no. 1-3, pp. 448-456, 1996.

[31] E. Benes, "Improved quartz crystal microbalance technique," Journal of Applied Physics, vol. 56, no. 3, pp. 608-626, 1984.

[32] K. Kanazawa, "Steady state and transient QCM solutions at the metal | solution interface," Journal of Electroanalytical Chemistry, vol. 524-525, pp. 103-109, 2002.

[33] K. Kanazawa, C. W. Frank, and J. Hardesty, "Resonances of soft films under liquid on the QCM," ECS Transactions, vol. 16, no. 11, pp. 419-429, 2008.

[34] N.-J. Cho, K. H. Cheong, C. Lee, C. W. Frank, and J. S. Glenn, "Binding dynamics of hepatitis C virus' NS5A amphipathic peptide to cell and model membranes," Journal of Virology, vol. 81, no. 12, pp. 6682-6689, 2007.

[35] N.-J. Cho, S.-J. Cho, K. H. Cheong, J. S. Glenn, and C. W. Frank, "Employing an amphipathic viral peptide to create a lipid bilayer on $\mathrm{Au}$ and $\mathrm{TiO}_{2}$," Journal of the American Chemical Society, vol. 129, no. 33, pp. 10050-10051, 2007.

[36] N.-J. Cho, J. N. D’Amour, J. Stalgren, W. Knoll, K. Kanazawa, and C. W. Frank, "Quartz resonator signatures under 
Newtonian liquid loading for initial instrument check," Journal of Colloid and Interface Science, vol. 315, no. 1, pp. 248-254, 2007.

[37] N.-J. Cho, K. Kanazawa, J. S. Glenn, and C. W. Frank, "Employing two different quartz crystal microbalance models to study changes in viscoelastic behavior upon transformation of lipid vesicles to a bilayer on a gold surface," Analytical Chemistry, vol. 79, no. 18, pp. 7027-7035, 2007.

[38] B. Kasemo, "Biological surface science," Surface Science, vol. 500, no. 1-3, pp. 656-677, 2002.

[39] C. A. Keller, K. Glasmastar, V. P. Zhdanov, and B. Kasemo, "Formation of supported membranes from vesicles," Physical Review Letters, vol. 84, no. 23, pp. 5443-5446, 2000.

[40] M. Rodahl, F. Hook, C. Fredriksson, et al., "Simultaneous frequency and dissipation factor QCM measurements of biomolecular adsorption and cell adhesion," Faraday Discussions, vol. 107, pp. 229-246, 1997.

[41] C. A. Keller and B. Kasemo, "Surface specific kinetics of lipid vesicle adsorption measured with a quartz crystal microbalance," Biophysical Journal, vol. 75, no. 3, pp. 1397 1402, 1998.

[42] F. Höök, B. Kasemo, T. Nylander, C. Fant, K. Sott, and H. Elwing, "Variations in coupled water, viscoelastic properties, and film thickness of a Mefp-1 protein film during adsorption and cross-linking: a quartz crystal microbalance with dissipation monitoring, ellipsometry, and surface plasmon resonance study," Analytical Chemistry, vol. 73, no. 24, pp. 5796-5804, 2001.

[43] M. K. Jain, A. Strickholm, F. P. White, and E. H. Cordes, "Electronic conduction across a black lipid membrane," Nature, vol. 227, no. 5259, pp. 705-707, 1970.

[44] W. A. Huemoeller and H. T. Tien, "A simple set-up for black lipid membrane experiments," Journal of Chemical Eduction, vol. 47, no. 6, pp. 469-470, 1970.

[45] L. K. Tamm and H. M. McConnell, "Supported phospholipid bilayers," Biophysical Journal, vol. 47, no. 1, pp. 105-113, 1985.

[46] H. M. McConnell, T. H. Watts, R. M. Weis, and A. A. Brain, "Supported planar membranes in studies of cell-cell recognition in the immune system," Biochimica et Biophysica Acta, vol. 864, no. 1, pp. 95-106, 1986.

[47] E. Sackmann, "Supported membranes: scientific and practical applications," Science, vol. 271, no. 5245, pp. 43-48, 1996.

[48] E. Sackmann and M. Tanaka, "Supported membranes on soft polymer cushions: fabrication, characterization and applications," Trends in Biotechnology, vol. 18, no. 2, pp. 58-64, 2000.

[49] D. Gidalevitz, Y. Ishitsuka, A. S. Muresan, et al., "Interaction of antimicrobial peptide protegrin with biomembranes," Proceedings of the National Academy of Sciences of the United States of America, vol. 100, no. 11, pp. 6302-6307, 2003.

[50] Y. Ishitsuka, D. Gidalevitz, A. J. Waring, R. I. Lehrer, and K. Y. Lee, "The selective interaction of protegrin-1 with a model cell membrane: the role of the lipid tail unsaturation," Biophysical Journal, vol. 84, p. 513a, 2003.

[51] M. Andersson, A. Sellborn, C. Fant, C. Gretzer, and H. Elwing, "Acoustics of blood plasma on solid surfaces," Journal of Biomaterials Science, Polymer Edition, vol. 13, no. 8, pp. 907917, 2002.

[52] R. J. Barfoot, K. H. Sheikh, B. R. G. Johnson, et al., "Minimal F-actin cytoskeletal system for planar supported phospholipid bilayers," Langmuir, vol. 24, no. 13, pp. 6827-6836, 2008.

[53] S. Belegrinou, I. Mannelli, P. Lisboa, et al., " $\mathrm{pH}$-dependent immobilization of proteins on surfaces functionalized by plasma-enhanced chemical vapor deposition of poly(acrylic acid)-and polyethylene oxide-like films," Langmuir, vol. 24, no. 14, pp. 7251-7261, 2008.

[54] S. Boujday, A. Bantegnie, E. Briand, P.-G. Marnet, M. Salmain, and C.-M. Pradier, "In-depth investigation of protein adsorption on gold surfaces: correlating the structure and density to the efficiency of the sensing layer," Journal of Physical Chemistry B, vol. 112, no. 21, pp. 6708-6715, 2008.

[55] H. Chen, X. Su, K. G. Neoh, and W.-S. Choe, "Contextdependent adsorption behavior of cyclic and linear peptides on metal oxide surfaces," Langmuir, vol. 25, no. 3, pp. 15881593, 2009.

[56] C. Fant, H. Elwing, and F. Hook, "The influence of crosslinking on protein-protein interactions in a marine adhesive: the case of two byssus plaque proteins from the blue mussel," Biomacromolecules, vol. 3, no. 4, pp. 732-741, 2002.

[57] R. Fogel, P. Mashazi, T. Nyokong, and J. Limson, "Critical assessment of the Quartz Crystal Microbalance with Dissipation as an analytical tool for biosensor development and fundamental studies: metallophthalocyanine-glucose oxidase biocomposite sensors," Biosensors and Bioelectronics, vol. 23, no. 1, pp. 95-101, 2007.

[58] M. S. Lord, M. H. Stenzel, A. Simmons, and B. K. Milthorpe, "The effect of charged groups on protein interactions with poly(HEMA) hydrogels," Biomaterials, vol. 27, no. 4, pp. 567$575,2006$.

[59] J. Malmstrom, H. Agheli, P. Kingshott, and D. S. Sutherland, "Viscoelastic modeling of highly hydrated laminin layers at homogeneous and nanostructured surfaces: quantification of protein layer properties using QCM-D and SPR," Langmuir, vol. 23, no. 19, pp. 9760-9768, 2007.

[60] W. Y. X. Peh, E. Reimhult, H. F. Teh, J. S. Thomsen, and X. Su, "Understanding ligand binding effects on the conformation of estrogen receptor $\alpha$-DNA complexes: a combinational quartz crystal microbalance with dissipation and surface plasmon resonance study," Biophysical Journal, vol. 92, no. 12, pp. 44154423, 2007. 

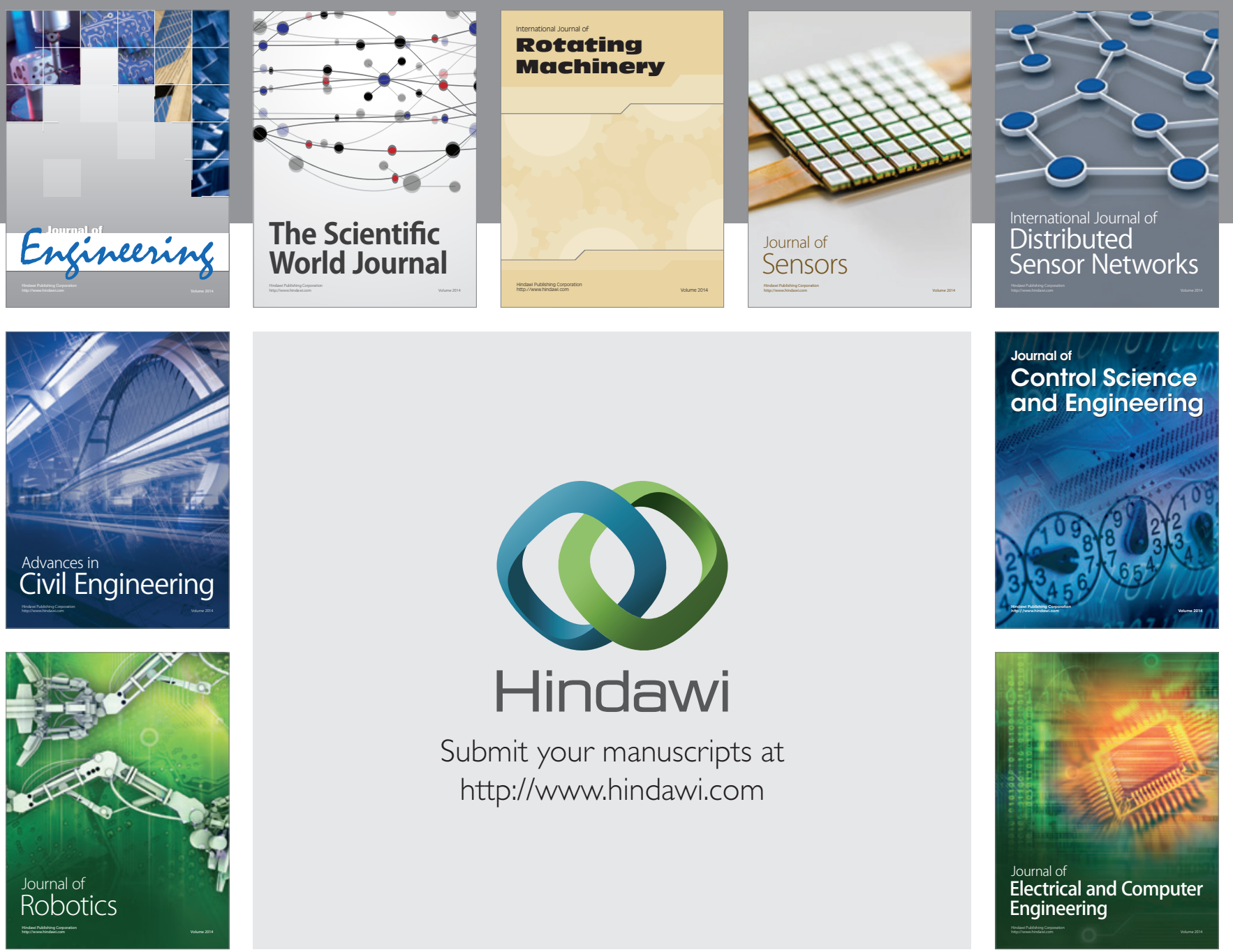

Submit your manuscripts at

http://www.hindawi.com
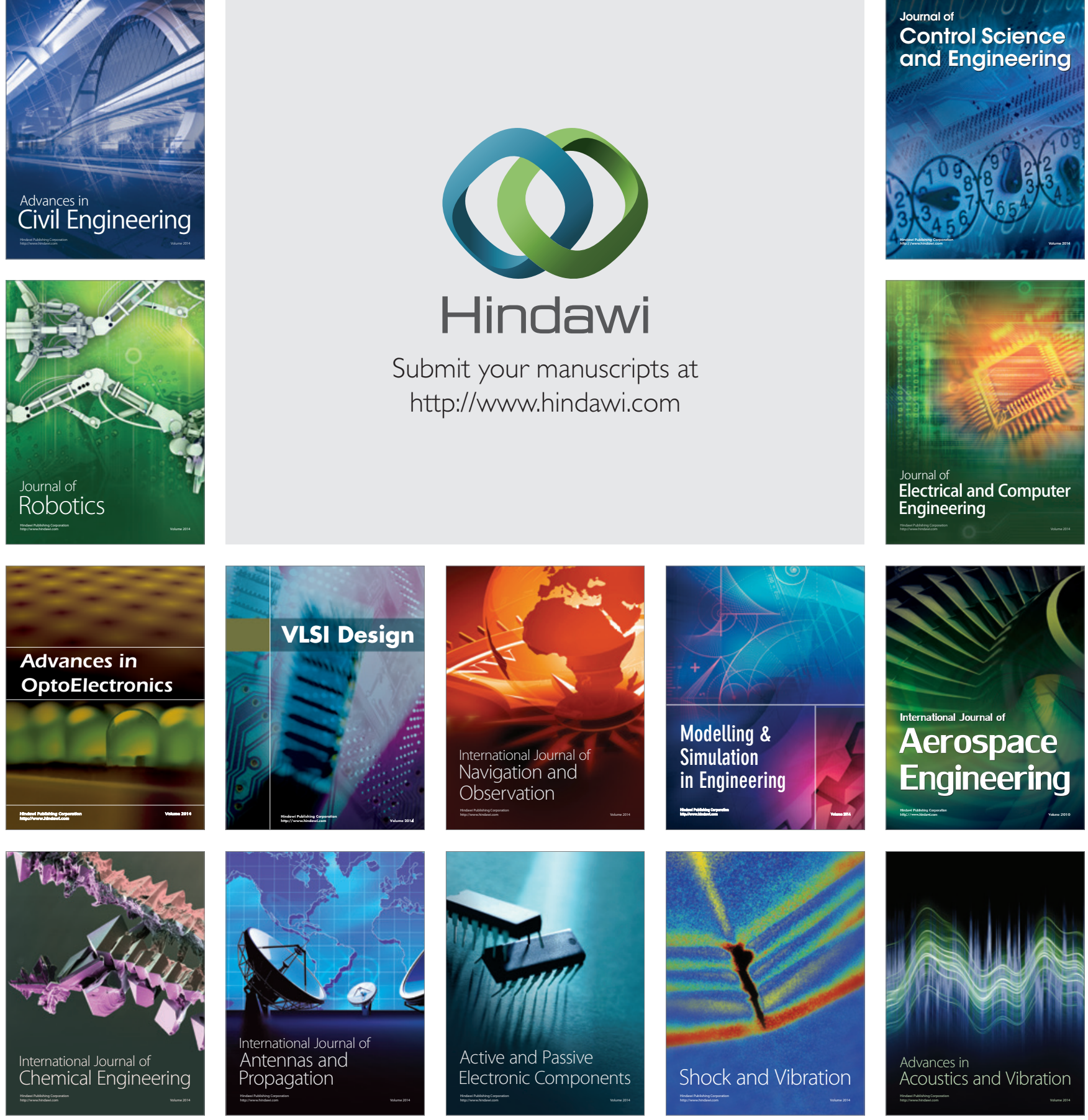